\title{
Nonadiabatic Molecular Dynamics on Graphics Processing Units: Performance and Application to Rotary Molecular Motors
}

\author{
Laurens D. M. Peters, ${ }^{\dagger}$ Jörg Kussmann, ${ }^{\dagger}$ and Christian Ochsenfeld $*,+\ddagger \odot$ \\ ${ }^{\dagger}$ Chair of Theoretical Chemistry, Department of Chemistry, University of Munich (LMU), Butenandtstr. 7, D-81377 München, \\ Germany \\ ${ }^{\ddagger}$ Max Planck Institute for Solid State Research, Heisenbergstr. 1, D-70569 Stuttgart, Germany
}

Supporting Information

ABSTRACT: Nonadiabatic molecular dynamics (NAMD) simulations of molecular systems require the efficient evaluation of excited-state properties, such as energies, gradients, and nonadiabatic coupling vectors. Here, we investigate the use of graphics processing units (GPUs) in addition to central processing units (CPUs) to efficiently calculate these properties at the time-dependent density functional theory (TDDFT) level of theory. Our implementation in the FermiONs++ program package uses the J-engine and a preselective screening procedure for the calculation of

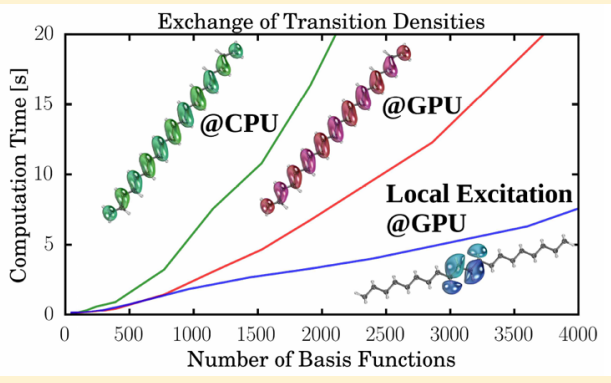

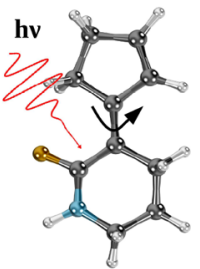

1 ps Simulation: 1 Day @ 4 GPUs Coulomb and exchange kernels, respectively. We observe good speed-ups for small and large molecular systems (comparable to those observed in ground-state calculations) and reduced (down to sublinear) scaling behavior with respect to the system size (depending on the spatial locality of the investigated excitation). As a first illustrative application, we present efficient NAMD simulations of a series of newly designed light-driven rotary molecular motors and compare their $S_{1}$ lifetimes. Although all four rotors show different $S_{1}$ excitation energies, their ability to rotate upon excitation is conserved, making the series an interesting starting point for rotary molecular motors with tunable excitation energies.

\section{INTRODUCTION}

Nonadiabatic processes such as electronic excitations, ${ }^{1}$ radiationless transitions, ${ }^{2}$ and electron transfer ${ }^{3}$ are of key importance in chemistry and biology. One of the most prominent examples in these fields is the rhodopsin protein, whose chromophore undergoes a photoisomerization when exposed to light. ${ }^{4}$ This energy conversion of light to mechanical motion has inspired chemists to design synthetic light-driven rotary molecular motors, ${ }^{5,6}$ for which Bernard Feringa was awarded the Nobel Prize in chemistry in 2016 together with Jean Pierre Sauvage and Sir James Fraser Stoddart. ${ }^{7}$ The description of theses nonadiabatic processes is an ongoing challenge in modern quantum chemistry (e.g., ref 8 ). This is mainly due to the fact that (1) excited states have to be taken into account and (2) the dynamics of the nuclei need to be considered.

To tackle the first challenge, several quantum-chemical methods have been developed. Examples include the complete active space self-consistent field (CASSCF) method, ${ }^{9}$ the algebraic-diagrammatic construction $(\operatorname{ADC}(2)),{ }^{10}$ several coupled cluster methods (e.g., CC2), ${ }_{11}^{11}$ and time-dependent density functional theory (TDDFT). ${ }^{12,13}$ The latter serves (despite its well-known limitations using today's functionals ${ }^{13,14}$ ) as a good compromise between effort and accuracy. ${ }^{15,16}$ As a consequence of this, not only excited-state energies but also excited-state gradients ${ }^{17}$ and nonadiabatic coupling vectors (NACVs) $)^{18-29}$ have been implemented to provide access to molecular properties at the TDDFT level of theory. Having excited-state energies and properties at hand, trajectory surface hopping $(\mathrm{TSH})^{30,31}$ is a straightforward way to conduct nonadiabatic molecular dynamics (NAMD) simulations including several electronic states.

Despite many advances in the field of $\mathrm{NAMD}^{32-34}$ and their broad field of application, ${ }^{35-44}$ it remains difficult or even impossible to investigate large molecular systems. The reason for this is that TSH requires, because of its stochastic nature, a large set of independent trajectories including many time steps, which involve expensive (even at the TDDFT level of theory) excited-state energy and property calculations. One way to accelerate NAMD simulations is the use of exciton models. ${ }^{45}$ Another approach is the use of graphics processing units (GPUs) in addition to central processing units (CPUs) for the calculations. It was shown that this leads to significant speedups for ground-state energy and forces evaluations ${ }^{46-53}$ and was also successfully applied to excited-state energies and properties $^{45,54-56}$ as well as ab initio multiple spawning ${ }^{57,58}$ and $\mathrm{NAMD}^{59,60}$ simulations.

In this work, we present efficient NAMD simulations with TDDFT energies, gradients, and NACVs calculated on hybrid

Received: August 29, 2019

Published: November 25, 2019 
CPU/GPU architectures using the FermiONs++ program package. $^{50-52}$ We start with a brief summary of the theory behind TSH as well as TDDFT energies, gradients, and NACVs in Section 2 and discuss their implementation on GPUs, featuring the hybrid CPU/GPU engine ${ }^{52}$ for the twoelectron integrals as well as the preselective screening procedure for the evaluation of exchange kernels. ${ }^{50,51}$ Computational details are given in Section 3. In Section 4, we discuss the accuracy and the performance of our GPUbased excited-state routines, investigating the scaling of our integral evaluations and contractions. We show timings for selected molecules containing more than 500 atoms. As a prototypical application, we investigate the photoinduced rotation of four newly designed rotary molecular motors via NAMD in Section 5, followed by a conclusion and an outlook.

\section{THEORY}

All equations in this section use the standard notation for orbitals: $i, j, k \ldots$ denote occupied, $a, b, c \ldots$ denote virtual, and $p$, $q, r \ldots$ denote arbitrary molecular orbitals, while $\mu, \nu, \lambda \ldots$ denote basis functions. $I, J, \ldots$ denote different electronic states. $v_{\mathrm{xc}}$ and $f_{\mathrm{xc}}$ are the first- and second-order exchange-correlation functional derivatives, respectively. $c_{\mathrm{x}}$ is the amount of exact exchange. $\mathbf{F}$ and $\mathbf{S}$ are the Kohn-Sham and the overlap matrices, respectively. $\mathbf{P}$ is the ground-state density, and $\mathbf{h}$ is the one-electron core Hamiltonian matrix. The two-electron integrals are written using Mulliken notation (..I..). Superscript $\xi$ denotes derivatives with respect to the nuclear coordinates, and $*$ and $\dagger$ symbolize complex conjugation and adjungation, respectively. For the sake of simplicity, we use the same symbol for matrices in the atomic orbital ( $\mathrm{AO})$ and molecular orbital (MO) bases with different indices (e.g., $P_{\mu \nu}$ vs $P_{p q}$ ).

2.1. Trajectory Surface Hopping. In nonadiabatic molecular dynamics (NAMD), the electronic time-dependent wave function of the system is assumed to be a linear combination of the time-independent electronic wave functions of the individual electronic states weighted by the state amplitudes $(\vec{c})$

$$
\Psi(\mathbf{x},\{\mathbf{r}\}, t)=\sum_{I} c_{I}(t) \Phi_{I}(\mathbf{x}(t),\{\mathbf{r}\})
$$

where $\mathbf{x}$ represents nuclear coordinates and $\{\mathbf{r}\}$ is the set of electronic coordinates. Having the state energies $\left(\omega_{I}\right.$, see Section 2.2) and nonadiabatic coupling vectors (NACVs, $\tau_{I \rightarrow J}^{\xi}$, see Section 2.4) at hand, it is possible to propagate the state amplitudes along with the nuclei during the molecular dynamics (MD) simulation using a unitary propagator: ${ }^{36}$

$$
\begin{gathered}
\vec{c}(t+\delta t) \approx \mathbf{U}(t, t+\delta t) \vec{c}(t) \\
\mathbf{U}(t, t+\delta t)=\exp \left(\frac{\mathbf{H}(t)+\mathbf{H}(t+\delta t)}{2} \delta t\right) \\
H_{I J}=\exp \left[\mathrm{i} \int_{0}^{t}\left(\omega_{I J}\right) \mathrm{dt}^{\prime}\right]\left(\dot{\mathbf{x}} \cdot \tau_{I \rightarrow J}^{\xi}\right)
\end{gathered}
$$

Here, $\omega_{I I}$ is the energy difference between two states $\left(\omega_{I}-\right.$ $\left.\omega_{I}\right)$. The applied $\delta t$ for the propagation of $\vec{c}$ is normally 3 orders of magnitude smaller than the time step of the MD simulation $(\Delta t) .^{31}$ In the fewest-switches surface hopping algorithm, ${ }^{30,31} \vec{c}$ is used to calculate the probability $g_{I \rightarrow J}^{t \rightarrow+\Delta t}$ of the system switching from its current state $I$ to another state $J$ at every time step:

$$
\begin{gathered}
g_{I \rightarrow J}^{t \rightarrow t+\Delta t}=-\frac{2 \Delta t}{a_{I I}} \int_{t}^{t+\Delta t} \operatorname{dt} \mathcal{R}\left\{a_{J I}^{*} H_{I I}\right\} \\
\mathbf{a}=\mathbf{c c}^{\dagger}
\end{gathered}
$$

If a random number between zero and one exceeds $g$, then the MD simulation is continued on the potential energy surface of state $J$ and the nuclear velocity is rescaled along $\tau_{I}^{\xi} \rightarrow$. Observables (e.g., lifetimes of states or relaxation pathways) can be drawn from ensembles of trajectories using a different set of random numbers. In the following sections, we will briefly summarize the calculation of the necessary ingredients of TSH (excited-state energies, gradients, and NACVs) at the time-dependent density functional theory (TDDFT) level of theory.

2.2. Excited-State Energies. Excitation energies can be calculated from linear response TDDFT by solving the TDDFT or the random phase approximation (RPA) equation $^{12,61,62}$

$$
\left[\begin{array}{cc}
\mathbf{A} & \mathbf{B} \\
\mathbf{B}^{*} & \mathbf{A}^{*}
\end{array}\right]\left(\begin{array}{l}
\mathbf{X}_{I} \\
\mathbf{Y}_{I}
\end{array}\right)=\omega_{I}\left[\begin{array}{cc}
\mathbf{1} & \mathbf{0} \\
\mathbf{0} & -\mathbf{1}
\end{array}\right]\left(\begin{array}{l}
\mathbf{X}_{I} \\
\mathbf{Y}_{I}
\end{array}\right)
$$

$\mathbf{A} \pm \mathbf{B}$ are the orbital rotation Hessians

$$
\begin{aligned}
& (\mathbf{A}+\mathbf{B})_{i a j b}=\delta_{i j} \delta_{a b}\left(\epsilon_{a}-\epsilon_{i}\right)+2(i a \mid j b)+2 f_{i a j b}^{\mathrm{xc}} \\
& \quad-c_{\mathrm{x}}[(j a \mid i b)+(a b \mid i j)] \\
& (\mathbf{A}-\mathbf{B})_{i a j b}=\delta_{i j} \delta_{a b}\left(\epsilon_{a}-\epsilon_{i}\right)+c_{\mathrm{x}}[(j a \mid i b)+(a b \mid i j)]
\end{aligned}
$$

with $\epsilon_{p}$ being the energy of orbital $p . \mathbf{X}_{I}$ and $\mathbf{Y}_{I}$ are the transition densities for excitation and de-excitation, respectively. Neglecting $\mathbf{B}$ is known as the Tamm-Dancoff approximation (TDA) of TDDFT, ${ }^{63}$ simplifying eq 4 to

$$
\mathbf{A X}_{I}=\omega_{I} \mathbf{X}_{I}
$$

Please note that eqs 4 and 7 are equivalent to the timedependent Hartree-Fock (TDHF) and configuration interaction singles (CIS) equations, respectively, when $c_{\mathrm{x}}$ is set equal to 1 and all exchange-correlation terms are neglected. TDA is computationally less demanding than TDDFT because fewer two-electron integrals have to be evaluated. It may also be more suitable for NAMD simulations because it is more stable (regarding its convergence) and thus typically delivers better results than TDDFT in the vicinity of conical intersections involving the ground state. ${ }^{39,64}$

TDDFT is nowadays widely used for the calculation of excitation energies, being a good compromise between accuracy and computational cost. ${ }^{15,16}$ Shortcomings are, however, the inability to calculate double excitations and the poor description of charge-transfer excitations. ${ }^{13,14}$ To tackle the latter, range-corrected functionals (e.g., $\omega \mathrm{B} 97^{65}$ ) have been introduced.

2.3. Excited-State Energy Gradients. To determine the energy gradient of an excited state $I$, one has to calculate the change in the excitation energy with respect to the nuclear coordinates $\left(\omega_{I}^{\xi}\right)$. The final equation in the $\mathrm{AO}$ basis has been derived by Furche et al. ${ }^{17}$

$$
\begin{aligned}
\omega_{I}^{\xi}= & \sum_{\mu \nu}\left\{h_{\mu \nu}^{\xi} P_{\mu \nu}^{I}-S_{\mu \nu}^{\xi} W_{\mu \nu}^{I}+v_{\mu \nu}^{x c(\xi)} P_{\mu \nu}^{I}\right\} \\
& +\sum_{\mu \nu \kappa \lambda}(\mu \nu \mid \kappa \lambda)^{\xi} \Gamma_{\mu \nu \kappa \lambda}^{I}+\sum_{\mu \nu \kappa \lambda} f_{\mu \nu \kappa \lambda}^{x c(\xi)} R_{\mu \nu}^{I} R_{\kappa \lambda}^{I}
\end{aligned}
$$


The calculations of the relaxed difference density matrix $\left(\mathbf{P}_{I}\right)$, the energy-weighted difference density matrix $\left(\mathbf{W}_{I}\right)$, and the two-particle difference density matrix $\left(\Gamma_{I}\right)$ are shown in the Appendix. The first two require an iterative solution of a $Z$ vector equation. ${ }^{17} \mathbf{R}_{I}$ is $\mathbf{X}_{I}+\mathbf{Y}_{I}$ or $\mathbf{X}_{I}$ in the case of TDDFT or TDA, respectively.

2.4. Nonadiabatic Coupling Vectors. The NACV between two states ( $I$ and $J$ ) is defined as

$$
\tau_{I \rightarrow J}^{\xi}=\left\langle\Phi_{I} \mid \Phi_{J}^{\xi}\right\rangle
$$

It thus describes the change of the overlap of the wave functions of $I$ and $J$ with respect to the nuclear coordinates. The first formulation of the NACV involving the ground state $\left(\tau_{0}^{\xi} \rightarrow I\right)$ derived by Chernyak and Mukamel ${ }^{18}$ was corrected for finite basis set effects by Send et al. ${ }^{23}$ in 2010:

$$
\begin{aligned}
\tau_{0 \rightarrow I}^{\xi}= & \sum_{\mu \nu}\left[h_{\mu \nu}^{\xi} P_{\mu \nu}^{0 I}-S_{\mu \nu}^{\xi} W_{\mu \nu}^{0 I}+v_{\mu \nu}^{x c(\xi)} P_{\mu \nu}^{0 I}\right] \\
& +\sum_{\mu \nu \kappa \lambda}(\mu \nu \mid \kappa \lambda)^{\xi} \Gamma_{\mu \nu \kappa \lambda}^{0 I}+\sum_{\mu \nu} S_{\mu \nu}^{[A] \xi} \gamma_{\mu \nu}^{0 I}
\end{aligned}
$$

Equations for the density matrices $\left(\mathbf{P}_{0 I}, \mathbf{W}_{0 I}\right.$, and $\left.\Gamma_{0 I}\right)$ are again given in the Appendix. They can be calculated directly from the TDDFT transition densities so that no $Z$-vector equation needs to be solved. $S_{\mu \nu}^{[A] \xi}$ denotes an antisymmetric overlap derivative

$$
S_{\mu \nu}^{[A] \xi}=\left\langle\chi_{\mu}^{\xi} \mid \chi_{\nu}\right\rangle-\left\langle\chi_{\mu} \mid \chi_{\nu}^{\xi}\right\rangle
$$

whereas $\gamma_{0 I}$ is defined as

$$
\gamma_{0 I}=\mathbf{L}_{I}
$$

with $\mathbf{L}_{I}$ being $\mathbf{X}_{I}-\mathbf{Y}_{I}$ or $\mathbf{X}_{I}$ in the case of TDDFT or TDA, respectively.

The calculation of NACVs between two excited states has been tackled by many publications showing expressions based on linear and quadratic response at different levels of theory. ${ }^{25,26,29,66-71}$ The main conclusion is that the use of quadratic response theory leads to unphysical poles when the energy difference between excited states matches the excitation energy of another state. ${ }^{66-70}$ The use of linear response theory or the so-called pseudowave function approach ${ }^{25-27,29}$ is therefore recommended. The resulting equation in the $\mathrm{AO}$ basis is as follows:

$$
\begin{aligned}
& \tau_{I \rightarrow J}^{\xi}=\frac{1}{\omega_{J I}}\left\{\sum_{\mu \nu}\left[h_{\mu \nu}^{\xi} P_{\mu \nu}^{I J}-S_{\mu \nu}^{\xi} W_{\mu \nu}^{I J}+v_{\mu \nu}^{\mathrm{xc}(\xi)} P_{\mu \nu}^{I J}\right]\right. \\
& \left.+\sum_{\mu \nu \kappa \lambda}(\mu \nu \mid \kappa \lambda)^{\xi} \Gamma_{\mu \nu \kappa \lambda}^{I J}+\sum_{\mu \nu \kappa \lambda} f_{\mu \nu \kappa \lambda}^{\mathrm{xc}(\xi)} R_{\mu \nu}^{I} R_{\kappa \lambda}^{J}\right\}+\sum_{\mu \nu} S_{\mu \nu}^{[A] \xi} \gamma_{\mu \nu}^{I J}
\end{aligned}
$$

Equations for the density matrices $\left(\mathbf{P}_{I J}, \mathbf{W}_{I J}\right.$, and $\left.\Gamma_{I J}\right)$ can again be found in the Appendix. $\gamma_{I J}$ is defined as

$$
\gamma_{I J}=\frac{1}{2}\left(\begin{array}{cc}
-\left(\mathbf{R}_{I}^{T} \mathbf{L}_{J}+\mathbf{L}_{I}^{T} \mathbf{R}_{J}\right)_{i j} & 0 \\
0 & \left(\mathbf{R}_{I} \mathbf{L}_{J}^{T}+\mathbf{L}_{I} \mathbf{R}_{J}^{T}\right)_{a b}
\end{array}\right)
$$

It was shown by Fatehi et al. ${ }^{25}$ that the antisymmetric overlap derivatives (eq 11) introduce translational variance into the NACV calculations. Neglecting these terms in eqs 10 and 13 is equivalent to adding electron-translational factors (ETFs).
However, trajectory surface hopping (TSH) simulations using NACVs with and without ETFs lead to nearly identical results for larger molecular systems. ${ }^{72}$

2.5. Graphics Processing Units. Graphics processing units (GPUs) significantly accelerate quantum-chemical calculations because they allow for an efficient evaluation and contraction of two-electron integrals (eq 15) and their derivatives with respect to the nuclear coordinates (eq 16)..$^{46-52}$

$$
\begin{aligned}
& J(\mathbf{M})_{\mu \nu}=\sum_{\kappa \lambda} M_{\kappa \lambda}(\mu \nu \mid \kappa \lambda) \\
& K(\mathbf{M})_{\mu \nu}=\sum_{\kappa \lambda} M_{\kappa \lambda}(\mu \kappa \mid \nu \lambda) \\
& J^{\xi}(\mathbf{N}, \mathbf{M})=\sum_{\mu \nu \kappa \lambda} N_{\mu \nu} M_{\kappa \lambda}(\mu \nu \mid \kappa \lambda)^{\xi} \\
& K^{\xi}(\mathbf{N}, \mathbf{M})=\sum_{\mu \nu \kappa \lambda} N_{\mu \nu} M_{\kappa \lambda}(\mu \kappa \mid \nu \lambda)^{\xi}
\end{aligned}
$$

$J$ and $K$ denote the Coulomb and exchange parts, respectively, and $\mathbf{M}$ and $\mathbf{N}$ denote general density matrices. For the Coulomb part, large speed-ups can be observed when the Jengine $^{73,74}$ is applied to the rearranged shell-pair data. ${ }^{47}$ To see comparable speed-ups for the exchange part, we apply an additional preselective screening (preLinK): ${ }^{50,51}$

$$
\begin{aligned}
& \sum_{\kappa \lambda} \sqrt{(\mu \kappa \mid \mu \kappa)} \times\left|M_{\kappa \lambda}\right| \times \sqrt{(\nu \lambda \mid \nu \lambda)} \geq \vartheta_{\text {pre }} \\
& K(\mathbf{M})_{\mu \nu} N_{\mu \nu} \geq \vartheta_{\text {pre }}^{\nabla}
\end{aligned}
$$

It determines the significant shell pairs (those with an expected value above the given thresholds $\vartheta_{\text {pre }}$ and $\vartheta_{\text {pre }}^{\nabla}$ ) before their distribution to and calculation on the GPUs. The two-electron integral evaluation can be done even more efficiently when the workload is spread among both GPUs and CPUs. ${ }^{52}$

In ground-state calculations, $\mathbf{M}$ and $\mathbf{N}$ are solely the groundstate density (P), whereas for excited states $\mathbf{M}$ and $\mathbf{N}$ can additionally be $\mathbf{P}_{I}, \mathbf{R}_{I}$, or $\mathbf{L}_{I}$. However, the discussed procedure (shell pair rearrangement, J-engine, and preLinK) is still valid and can easily be adapted to excited-state routines when keeping in mind that $\mathbf{M}$ is not always equal to $\mathbf{N}$ and may be nonsymmetric.

\section{COMPUTATIONAL DETAILS}

3.1. General Remarks. The FermiONs++ program package $^{50-52}$ was used for all calculations presented in this work. It was compiled using the Intel compiler (2019), ${ }^{75}$ and the Intel Math Kernel Library (MKL). Routines on AMD GPUs were compiled with the AMD APPSDK compiler. In addition, LibXC library v4.0.1 ${ }^{76,77}$ was used. In all calculations, we used the gm5 grid $^{78}$ (with the modified Becke weighting scheme described in ref 78) and tight thresholds for the SCF convergence $\left(\vartheta_{\mathrm{SCF}}=10^{-7}\right.$ using the FP-commutator), the integrals $\left(\vartheta_{\mathrm{INT}}=10^{-10}\right)$, and the $Z$-vector equation convergence $\left(\vartheta_{Z}=10^{-5}\right)$. Throughout all calculations, we neglected the symmetry of the molecules and solely calculated singlet excitations. Excited-state energies, gradients, and nonadiabatic coupling vectors (with electron transition factors) at the TDDFT level of theory were calculated using eqs 4,8 , 10 , and 13 , respectively. 
Table 1. Mean Absolute Errors (MAE, in Atomic Units) of Excitation Energies $\left(\omega_{I}\right)$, Gradients $\left(\omega_{I}^{\xi}\right)$, and Nonadiabatic Coupling Vectors $\left(\tau_{I \rightarrow I}\right)$ of Protonated Formaldimine (I) and the Schiff Base of Retinal (II) Calculated at the PBE0/def2-SVP Level of Theory on GPUs, Employing the preLinK Scheme and Using Different Thresholds for preLinK $\left(\vartheta_{\text {pre }}\right)$, the preLinK Gradient $\left(\vartheta_{\text {pre }}^{\nabla}\right)$, and the TDDFT Convergence $\left(\vartheta_{\text {TDDFT }}\right)^{a}$

\begin{tabular}{|c|c|c|c|c|c|c|c|c|}
\hline \multicolumn{9}{|c|}{ screening thresholds and convergence criteria } \\
\hline$\vartheta_{\text {pre }}$ & $10^{-3}$ & $10^{-4}$ & $10^{-4}$ & $10^{-5}$ & $10^{-5}$ & $10^{-5}$ & $10^{-5}$ & $10^{-5}$ \\
\hline$\vartheta_{\text {pre }}^{\nabla}$ & $10^{-11}$ & $10^{-11}$ & $10^{-11}$ & $10^{-11}$ & $10^{-11}$ & $10^{-9}$ & $10^{-10}$ & $10^{-11}$ \\
\hline$\vartheta_{\text {TDDFT }}$ & $10^{-5}$ & $10^{-5}$ & $10^{-6}$ & $10^{-6}$ & $10^{-7}$ & $10^{-7}$ & $10^{-7}$ & $10^{-7}$ \\
\hline \multicolumn{9}{|c|}{ I } \\
\hline $\operatorname{MAE}\left(\omega_{I}\right)$ & $2.2 \times 10^{-4}$ & $2.2 \times 10^{-4}$ & $8.8 \times 10^{-5}$ & $8.8 \times 10^{-5}$ & $2.3 \times 10^{-6}$ & $2.3 \times 10^{-6}$ & $2.3 \times 10^{-6}$ & $2.3 \times 10^{-6}$ \\
\hline $\operatorname{MAE}\left(\omega_{2}^{\xi}\right)$ & $5.8 \times 10^{-5}$ & $5.8 \times 10^{-5}$ & $3.7 \times 10^{-5}$ & $3.7 \times 10^{-5}$ & $6.5 \times 10^{-6}$ & $6.7 \times 10^{-6}$ & $6.6 \times 10^{-6}$ & $6.5 \times 10^{-6}$ \\
\hline $\operatorname{MAE}\left(\tau_{0 \rightarrow 1}\right)$ & $5.7 \times 10^{-4}$ & $5.7 \times 10^{-4}$ & $1.2 \times 10^{-4}$ & $1.2 \times 10^{-4}$ & $9.2 \times 10^{-5}$ & $8.8 \times 10^{-5}$ & $9.1 \times 10^{-5}$ & $9.2 \times 10^{-5}$ \\
\hline $\operatorname{MAE}\left(\tau_{1 \rightarrow 2}\right)$ & $1.0 \times 10^{-3}$ & $9.1 \times 10^{-4}$ & $4.7 \times 10^{-4}$ & $4.7 \times 10^{-4}$ & $2.8 \times 10^{-4}$ & $9.2 \times 10^{-4}$ & $5.5 \times 10^{-4}$ & $2.8 \times 10^{-4}$ \\
\hline \multicolumn{9}{|c|}{ II } \\
\hline $\operatorname{MAE}\left(\omega_{I}\right)$ & $1.9 \times 10^{-4}$ & $1.9 \times 10^{-4}$ & $9.6 \times 10^{-5}$ & $9.6 \times 10^{-5}$ & $1.1 \times 10^{-5}$ & $1.1 \times 10^{-5}$ & $1.1 \times 10^{-5}$ & $1.1 \times 10^{-5}$ \\
\hline $\operatorname{MAE}\left(\omega_{2}^{\xi}\right)$ & $2.8 \times 10^{-5}$ & $2.8 \times 10^{-5}$ & $1.2 \times 10^{-5}$ & $1.2 \times 10^{-5}$ & $3.1 \times 10^{-6}$ & $3.8 \times 10^{-6}$ & $3.2 \times 10^{-6}$ & $3.1 \times 10^{-6}$ \\
\hline $\operatorname{MAE}\left(\tau_{0 \rightarrow 1}\right)$ & $1.7 \times 10^{-3}$ & $1.7 \times 10^{-3}$ & $7.5 \times 10^{-4}$ & $7.5 \times 10^{-4}$ & $2.0 \times 10^{-4}$ & $2.0 \times 10^{-4}$ & $2.0 \times 10^{-4}$ & $2.0 \times 10^{-4}$ \\
\hline $\operatorname{MAE}\left(\tau_{1 \rightarrow 2}\right)$ & $8.9 \times 10^{-4}$ & $8.9 \times 10^{-4}$ & $3.8 \times 10^{-4}$ & $3.8 \times 10^{-4}$ & $1.1 \times 10^{-4}$ & $1.2 \times 10^{-4}$ & $1.1 \times 10^{-4}$ & $1.1 \times 10^{-4}$ \\
\hline
\end{tabular}

${ }^{a}$ A calculation on CPUs with $\vartheta_{\mathrm{TDDFT}}=10^{-7}$ and without CFMM or preLinK is used as a reference. Throughout this work, we will use accurate $\left(\vartheta_{\mathrm{pre}}=10^{-3}, \vartheta_{\mathrm{pre}}^{\nabla}=10^{-10}\right.$, and $\left.\vartheta_{\mathrm{TDDFT}}=10^{-5}\right)$ or tight thresholds $\left(\vartheta_{\mathrm{pre}}=10^{-4}, \vartheta_{\mathrm{pre}}^{\nabla}=10^{-10}\right.$, and $\left.\vartheta_{\mathrm{TDDFT}}=10^{-6}\right)$.

3.2. Preparation and Calculation of Systems I, II, III $\mathrm{IV}_{\mathrm{n}}, \mathrm{V}, \mathrm{VI}$, and VII. To illustrate the performance and/or accuracy of our implementation on GPUs, we use protonated formaldimine (I), the Schiff base of retinal (II), a series of linear polyethynes $\left(\mathbf{I I I}_{n}\right)$ and dialkylethenes $\left(\mathbf{I V}_{n}\right)$, a motorized nanocar (without "wheels", V), ${ }^{79}$ and one (VI) and three (VII) pores of a covalent organic framework ${ }^{80}$ as example molecules. The structures of I, II, and $\mathbf{V}$ have been optimized at the $\mathrm{PBE0}^{81-84} /$ def2-SVP ${ }^{85,86}$ level of theory, while III $_{n}$ and $\mathbf{I V}_{n}$ have not been optimized to maintain their linear structures. VI and VII have been prepared according to ref 80 . All structures are available at https://www.cup.uni-muenchen.de/ pc/ochsenfeld/download/.

TDDFT energies and properties of I, II, III ${ }_{n}, \mathbf{I V}_{n}, \mathbf{V}, \mathbf{V I}$, and VII were calculated at the PBE0/def2-SVP or PBE/def2-TZVP level of theory. We used tight thresholds for preLinK $\left(\vartheta_{\text {pre }}=\right.$ $\left.10^{-4}\right)$, the preLinK gradient $\left(\vartheta_{\text {pre }}^{\nabla}=10^{-10}\right)$, and the TDDFT convergence $\left(\vartheta_{\text {TDDFT }}=10^{-6}\right)$. For I, II, III ${ }_{n}, \mathbf{I V}_{n}, \mathbf{V}$, VI, and VII, four, six, five, three, six, seven, and seven states were taken into account, respectively.

When investigating the accuracy, we use a calculation performed on CPUs with tight thresholds $\left(\vartheta_{\text {TDDFT }}=10^{-7}\right)$ as a reference. To allow for a fair comparison, we employed the continuous fast multipole method (CFMM) $)^{87,88}$ and the LinK scheme $^{89,90}$ for Coulomb and exchange kernels (and their derivatives) on CPUs when comparing CPU and GPU performance. Coulomb and exchange kernels on GPUs were calculated with the J-engine ${ }^{73,74}$ and the preLinK scheme, ${ }^{50,51}$ respectively. Timings of integral evaluations and entire routines were determined as an average over five independent calculations on two Intel Xeon CPU E5 2640 v4@2.20 $\mathrm{GHz}$ (20 threads) CPUs and four AMD FirePro 3D W8100 GPUs. The scaling behavior is determined as the slope of the corresponding log-log plots (Supporting Information) using the timings of III $_{n}$ and IV $_{n}$ with $n=40,50,75,100$. The parallel efficiency is determined as the ratio between the measured and ideal speed-ups.

3.3. Preparation and Calculation of the Rotary Molecular Motors. The structures of the four rotary molecular machines (C, N, S, and $\mathbf{O}$ ) have been optimized at the $\omega \mathrm{B} 97^{65} / \mathrm{def} 2$-SVP level of theory. Excited-state properties and timings were calculated at the TDA ( $\omega$ B97) def2-SVP) level of theory using accurate thresholds $\left(\vartheta_{\text {pre }}=\right.$ $10^{-3}, \vartheta_{\text {pre }}^{\nabla}=10^{-10}$, and $\left.\vartheta_{\text {TDDFT }}=10^{-5}\right)$. NAMD simulations were conducted at the same level of theory. The propagation of the nuclei was calculated using the Velocity Verlet algorithm. $^{91,92}$ The extended Lagrangian method $^{93}$ for the extrapolation of ground-state density was used to accelerate SCF convergence. Transition densities and relaxed difference densities of the previous step were used as guesses for the TDA and $Z$-vector equation, respectively.

Twenty initial geometries and velocities (available at https://www.cup.uni-muenchen.de/pc/ochsenfeld/download/ ) were drawn from a 5 ps ground-state NVT simulation (200 fs equilibration, 0.2 fs step size, velocity rescaling thermostat ${ }^{94}$ ) at the same level of theory. From each initial condition, five independent (different series of random numbers) NAMD simulations were conducted for 1 ps ( $0.2 \mathrm{fs}$ step size) without equilibration, thermostat, or decoherence correction, starting at the first excited singlet state $\left(S_{1}\right)$. At every step of the simulation, the overall rotation and translation of the molecule was removed. Three excited states were taken into account, whereas only the coupling vector from the ground state to the first excited state was calculated and used for the propagation of the state amplitudes (eq 2) and the calculation of the hopping probability (eq 3 ).

\section{PERFORMANCE}

4.1. Accuracy and Thresholds. We start with an analysis of the errors of excited-state energies and properties introduced by their calculation on GPUs and the use of the preLinK scheme ${ }^{51,52}$ for integrals and integral derivatives.

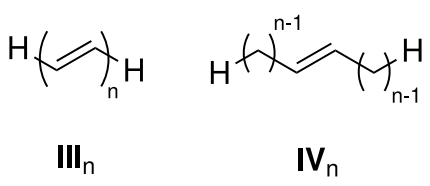

Figure 1. Structures of the linear polyethynes $\left(\mathrm{III}_{n}\right)$ and dialkylethenes $\left(\mathrm{IV}_{n}\right)$. 
(a)

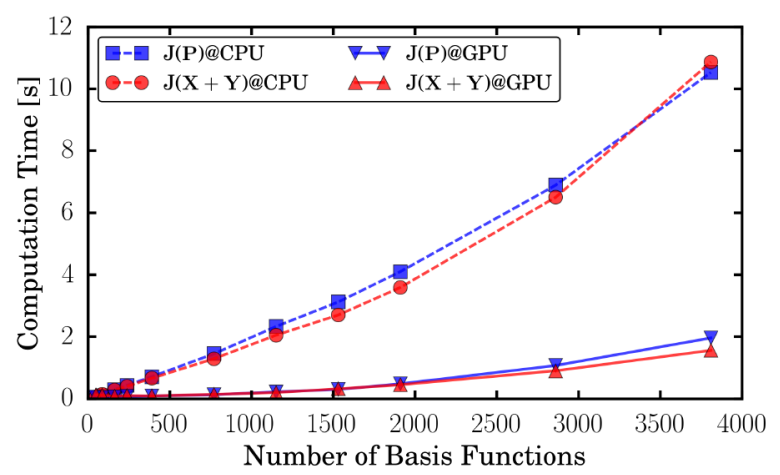

(c)

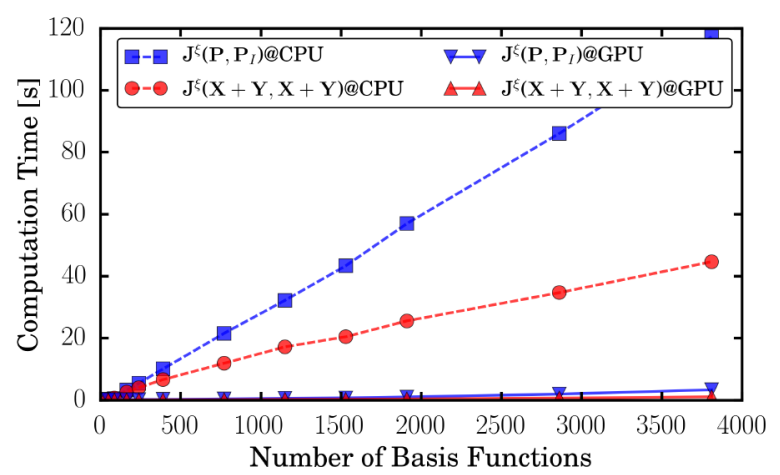

(b)

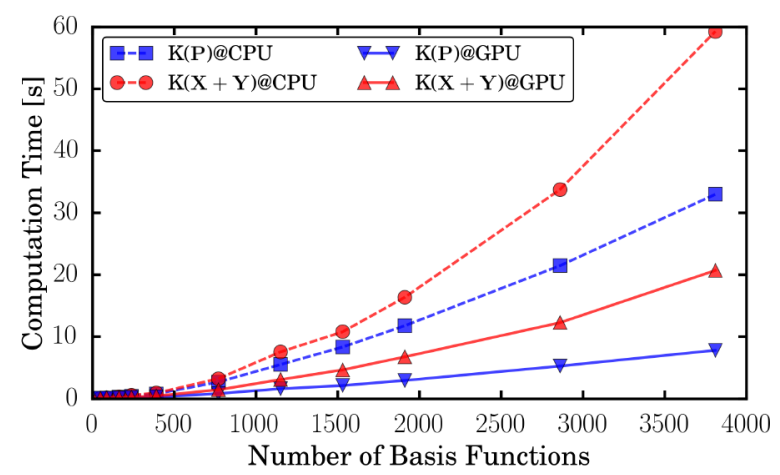

(d)

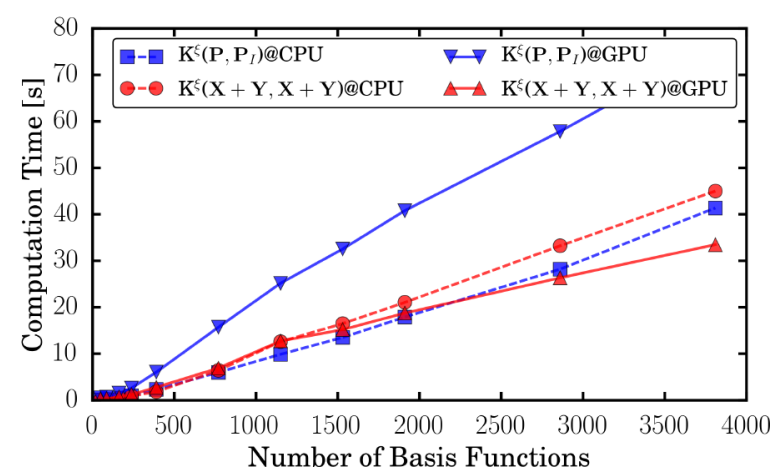

Figure 2. Timings of (a, c) Coulomb and (b, d) exchange integral evaluations ( $a$, b; eq 15) and their derivatives with respect to the nuclear coordinates (c, d; eq 16) of polyethyne ( $\mathrm{III}_{n}$ ) with $n=1-100$ calculated at the PBE0/def2-SVP level of theory on CPUs (dashed lines) and GPUs (solid lines). For details on the calculations and the computational setup, see Sections 3.1 and 3.2.

Therefore, we compare calculations of the smallest Schiff base (protonated formaldimine, $\mathrm{CH}_{2} \mathrm{NH}_{2}^{+}, \mathrm{I}$ ) and the Schiff base of retinal $\left(\mathrm{C}_{20} \mathrm{H}_{30} \mathrm{~N}^{+}\right.$, II $)$with GPUs and preLinK to calculations on CPUs without prescreening or the continuous fast multipole method (CFMM). (For computational details, see Section 3.2.) The mean absolute errors for different thresholds are listed in Table 1.

The choice of the preLinK threshold $\left(\vartheta_{\text {pre }}\right)$ for excited-state calculations should always depend on the applied convergence threshold for the TDDFT equation $\left(\vartheta_{\text {TDDFT }}\right)$. If the chosen threshold is too loose, then the iterative solution of the TDDFT equation does not converge (especially for large molecular systems), while a $\vartheta_{\text {pre }}$ that is too tight does not improve the result when $\vartheta_{\text {TDDFT }}$ is not adjusted accordingly (Table 1). The latter is also attributed to the small effect of preLinK on these relatively small systems. ${ }^{50,51}$ In our calculations with the FermiONs++ program package, the "ideal" $\vartheta_{\text {pre }}$ is 2 orders of magnitude larger than $\vartheta_{\text {TDDFT. }}$ A tightening of these two parameters (left side of Table 1) systematically leads to smaller errors. However, the errors in the coupling vectors of the large system do not fall below $10^{-4}$ a.u., marking the numerical limit of these second- to thirdorder properties. The use of the tight thresholds $\left(\vartheta_{\mathrm{TDDFT}}=\right.$ $10^{-6}, \vartheta_{\text {pre }}=10^{-4}$ ) should thus, in general, be sufficient because all errors are below $10^{-3}$ a.u. A looser preLinK threshold $\left(\vartheta_{\text {pre }}\right.$ $=10^{-3}$ a.u.), which is expected to give $\mu \mathrm{H}$ accuracy for groundstate properties, is still accurate for excited-state properties and could be used in extensive application calculations such as NAMD simulations, where observables are determined as ensemble averages.
The effect of the preLinK gradient threshold $\left(\vartheta_{\text {pre }}^{\nabla}\right)$ on the error is not straightforward (right side of Table 1). However, we recommend it to be at least as tight as the integral threshold $\left(\vartheta_{\text {INT }}=10^{-10}\right)$. Because the difference between $\vartheta_{\text {pre }}^{\nabla}=10^{-11}$ and $\vartheta_{\text {pre }}^{\nabla}=10^{-10}$ is negligibly small, we apply the latter throughout this work.

4.2. Scaling with the System Size. To compare timings on CPUs and GPUs and to investigate the effective scaling behavior of the excited-state integral routines on GPUs, we use integral timings of linear polyethynes ( III $\left._{n}\right)$ and dialkylethenes $\left(\mathbf{I V}_{n}\right)$ (for structures, see Figure 1). In Figure 2, we compare CPU and GPU timings of Coulomb (Figure 2a,c) and exchange (Figure 2b,d) calculations at the PBE0/def2-SVP level of theory. We show contractions of the ground-state density $(J / K(\mathbf{P}))$, the transition density of the first excited state $\left(J / K\left(\mathbf{X}_{1}+\mathbf{Y}_{1}\right)\right)$ (Figure $\left.2 \mathrm{a}, \mathrm{b}\right)$, the integral derivatives involving the ground state and the relaxed difference density of the first excited state $\left(J^{\xi} / K^{\xi}\left(\mathbf{P}, \mathbf{P}_{1}\right)\right)$, and the transition density of the first excited state $\left(J^{\xi} / K^{\xi}\left(\mathbf{X}_{1}+\mathbf{Y}_{1}, \mathbf{X}_{1}+\mathbf{Y}_{1}\right)\right.$ ) (Figure $2 \mathrm{c}, \mathrm{d})$. The same integrals are shown in Figure 3 , where we compare the timings of $\mathbf{I I I}_{n}$ and $\mathbf{I V}_{n}$ on GPUs. The effective scaling behaviors of the integral evaluations are shown in the Supporting Information. Definitions of the integral contractions are given in eqs 15 and 16. Details of the calculations are given in Sections 3.1 and 3.2.

For the Coulomb and exchange kernels of the transition densities, we observe similar speed-ups (up to a factor of 5) as for the ground-state kernels (Figure 2a,b). The accelerations of the gradient kernels stem (nearly exclusively) from their Coulomb part, which is extremely efficient on GPUs (Figures 
(a)

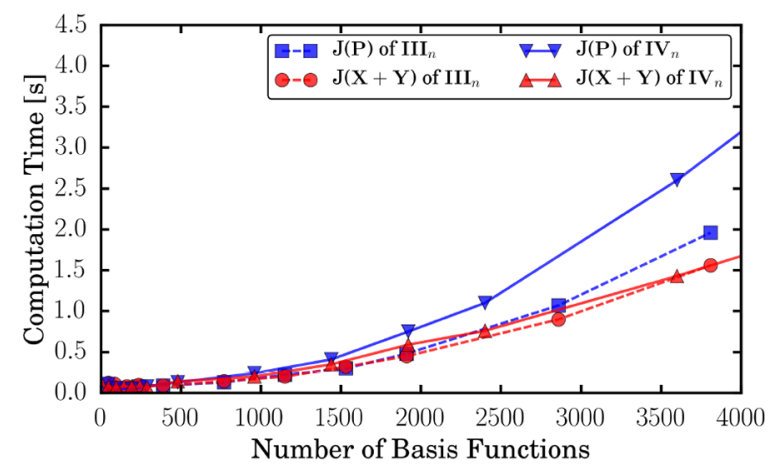

(c)

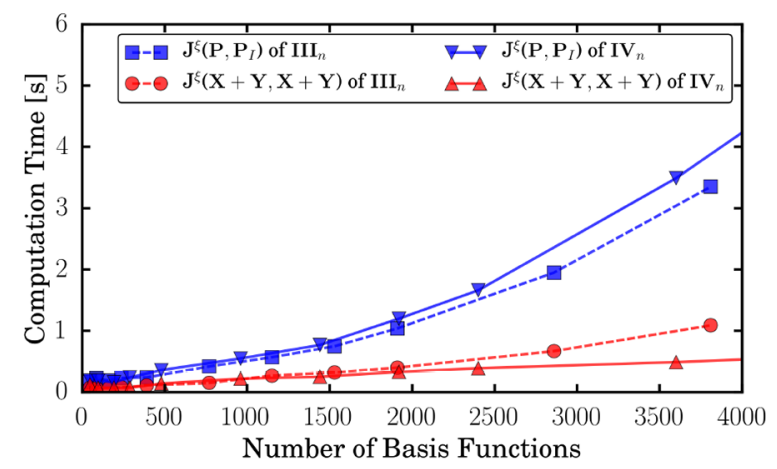

(b)

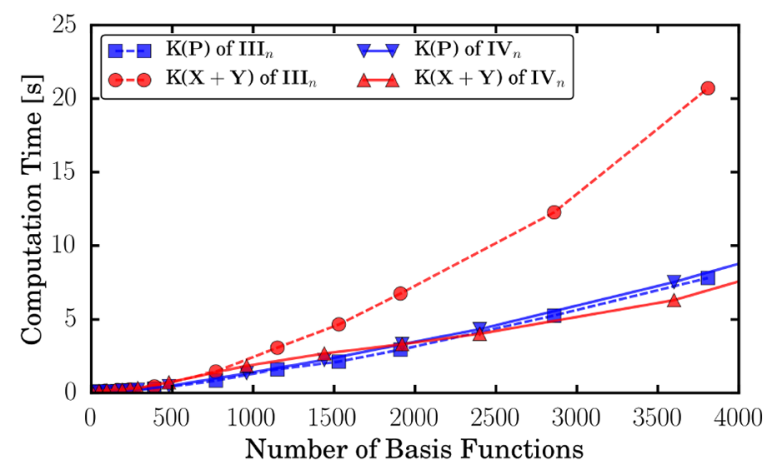

(d)

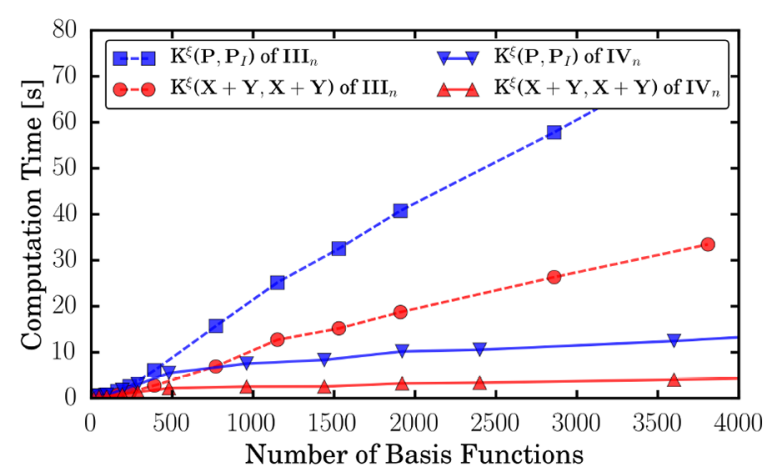

Figure 3. Timings of $(a, c)$ Coulomb and $(b, d)$ exchange integral evaluations $(a, b$; eq 15) and their derivatives with respect to the nuclear coordinates (c, d; eq 16) of polyethine ( $\mathrm{III}_{n}$, solid line) and dialkylethene $\left(\mathrm{IV}_{n}\right.$, dashed line) with $n=1-100$ calculated at the PBE0/def2-SVP level of theory on GPUs. For details on the calculations and the computational setup, see Sections 3.1 and 3.2.

2c). We also see minor speed-ups for $K^{\xi}\left(\mathbf{X}_{1}+\mathbf{Y}_{1}, \mathbf{X}_{1}+\mathbf{Y}_{1}\right)$ (Figures $2 \mathrm{~d})$. The evaluation of $K^{\xi}\left(\mathbf{P}, \mathbf{P}_{1}\right)$ is significantly slower because the GPU routine has to be called twice. This is due to the fact that the analytical exchange evaluation on GPUs $^{48-51}$ does not exploit the full symmetry of the twoelectron integral. Please note that this problem can be solved by applying a seminumerical exchange scheme, which is currently developed for GPUs in our group. ${ }^{95}$ For the largest molecule (III $\left.{ }_{100}\right)$, a speed-up of two for the entire determination of an excited-state gradient involving calculations of the ground-state and excited-state energy and gradient is observed. Here, it should be stressed that the linear algebra and the evaluation of the exchange-correlation kernels are performed entirely on CPUs.

When comparing the effective scaling behavior of the Coulomb integrals of III $_{n}$ (Supporting Information), one can see that the scaling of the GPU integrals is slightly larger than the scaling of the CPU integrals. This is due to the formal quadratic scaling of the J-engine employed on GPUs, in comparison to the (asymptotically linear scaling) CFMM method. This larger scaling behavior is, however, irrelevant as the prefactor of the routines is greatly reduced (Figure 2a,c). The scaling of the exchange integrals is slightly reduced by exploiting the preLinK method on GPUs. We observe $\sim 1.5$ and $\sim 1.0$ scalings for the integrals and the integral derivatives of these system sizes, respectively.

The excitation in III $_{n}$ is delocalized over the entire molecule, leading to similar scaling behavior for the ground-state and excited-state properties. To show the performance of a system with local excitation, we also investigate $\mathbf{I V}_{n}$, where only one double bond instead of a conjugated system is excited. This leads to massive speed-ups (Figure 3) of the excited-state exchange integrals and reduces their scaling significantly (Supporting Information). For the exchange integral derivatives, we even observe sublinear scaling behavior as a result of the preLinK screening. The effect on the Coulomb integrals is smaller, mainly because of the fact that these routines take only a few seconds even for the largest investigated molecules.

4.3. Example Calculations. To demonstrate the applicability of our excited-state properties routines in the FermiONs++ program package, ${ }^{50-52}$ we study four molecules of interest in modern excited-state research and show their timings at the PBE0/def2-SVP and PBE/def2-TZVP levels of theory on GPUs (Table 2). We investigate the Schiff base of retinal (II), a model system of the chromophore in rhodopsin, ${ }^{4}$ a nanocar ${ }^{79}(\mathrm{~V})$ using a rotary molecular motor, and one (VI) and three (VII) pores of a covalent organic framework, ${ }^{80}$ which catalyze the formation of hydrogen from water when exposed to light. Details of the calculations and the computational setup are again listed in Sections 3.1 and 3.2.

Table 2 shows that with the presented implementation on GPUs, excited-state properties and dynamics become accessible even for large systems and when applying DFT methods with exact exchange (e.g., PBE0) or triple- $\zeta$ basis sets. In our examples, the calculations of excited-state properties at the PBE/def2-TZVP level of theory are even faster than at the PBE0/def2-SVP level of theory mainly due to the fast Coulomb contractions discussed above. The only exception is $t\left(\omega_{1}^{\xi}\right)$ of VII, for which the Z-vector equation converges 
Table 2. Molecular Structures and Computational Times $(t(s))$ of the Schiff Base of Retinal (II), a Motorized Nanocar (V), and One (VI) and Three (VII) Pores of a Covalent Organic Framework, Calculating Ground-State Energies $\left(E_{0}\right)$ and Gradients $\left(E_{0}^{\xi}\right)$, Excited-State Energies $\left(\omega_{I}\right)$ and Gradients $\left(\omega_{I}^{\xi}\right)$, and Nonadiabatic Coupling Vectors $\left(\tau_{I \rightarrow J}\right)$ at the PBE0/def2SVP and PBE/def2-TZVP Levels of Theory on GPUs ${ }^{a}$

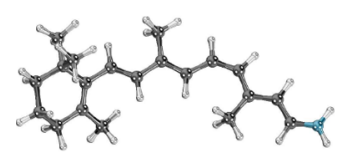

II

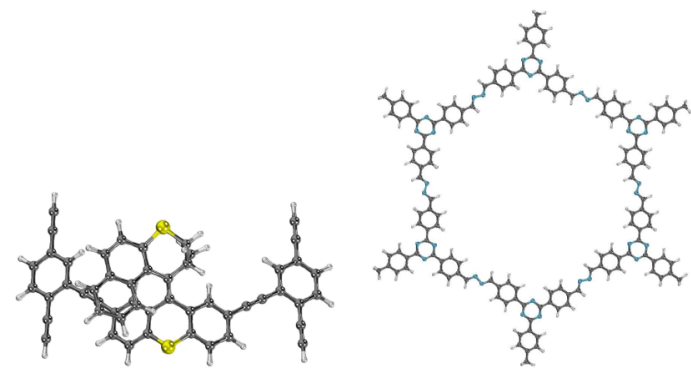

V

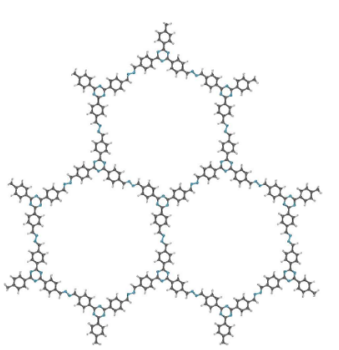

VII

\begin{tabular}{|c|c|c|c|c|}
\hline & II & V & VI & VII \\
\hline formula & $\mathrm{C}_{20} \mathrm{H}_{30} \mathrm{~N}^{+}$ & $\mathrm{C}_{51} \mathrm{H}_{28} \mathrm{~S}_{2}$ & $\mathrm{C}_{144} \mathrm{H}_{102} \mathrm{~N}_{30}$ & $\mathrm{C}_{312} \mathrm{H}_{213} \mathrm{~N}_{69}$ \\
\hline$N_{\text {atoms }}$ & 51 & 81 & 276 & 594 \\
\hline \multicolumn{5}{|c|}{ PBE0/def2-SVP } \\
\hline$t\left(E_{0}\right) /$ step & 1.3 & 4.4 & 17.8 & 67.7 \\
\hline$t\left(\omega_{1}\right) /($ step and state $)$ & 1.6 & 6.3 & 34.4 & 179.5 \\
\hline$t\left(E_{0}^{\xi}\right)$ & 12.36 & 54.5 & 140.9 & 334.3 \\
\hline$t\left(\omega_{1}^{\xi}\right)$ & 65.4 & 247.9 & 668.4 & 1392.6 \\
\hline$t\left(\tau_{0 \rightarrow 1}\right)$ & 24.3 & 123.4 & 375.2 & 914.8 \\
\hline$t\left(\tau_{1 \rightarrow 2}\right)$ & 82.7 & 349.4 & 959.0 & 1858.0 \\
\hline \multicolumn{5}{|c|}{ PBE/def2-TZVP } \\
\hline$t\left(E_{0}\right) /$ step & 1.8 & 1.5 & 51.5 & 272.6 \\
\hline$t\left(\omega_{1}\right) /($ step and state $)$ & 2.1 & 1.7 & 30.2 & 95.3 \\
\hline$t\left(E_{0}^{\xi}\right)$ & 6.0 & 5.2 & 65.3 & 241.9 \\
\hline$t\left(\omega_{1}^{\xi}\right)$ & 52.5 & 47.9 & 576.7 & 1942.9 \\
\hline$t\left(\tau_{0 \rightarrow 1}\right)$ & 12.3 & 10.1 & 124.4 & 377.0 \\
\hline$t\left(\tau_{1 \rightarrow 2}\right)$ & 56.1 & 52.7 & 523.2 & 1060.3 \\
\hline
\end{tabular}

${ }^{a}$ Please note that $t\left(E_{0}\right)$ and $t\left(\omega_{1}\right)$ are given per step (and state). For details on the calculations and the computational setup, see Sections 3.1 and 3.2 .

(a)

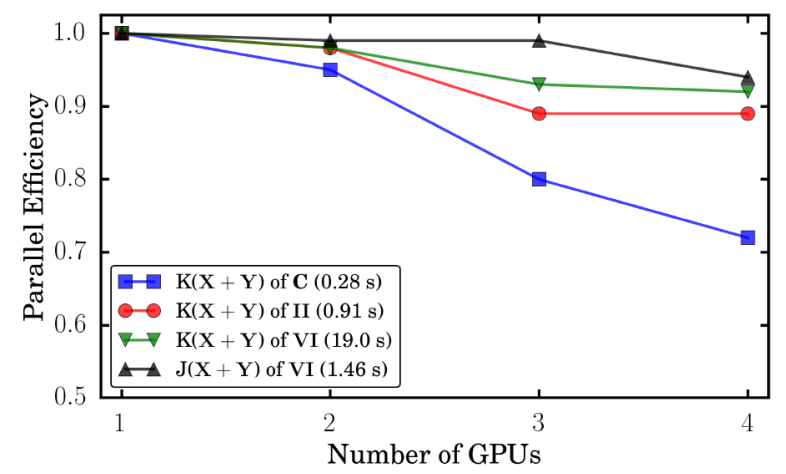

(b)

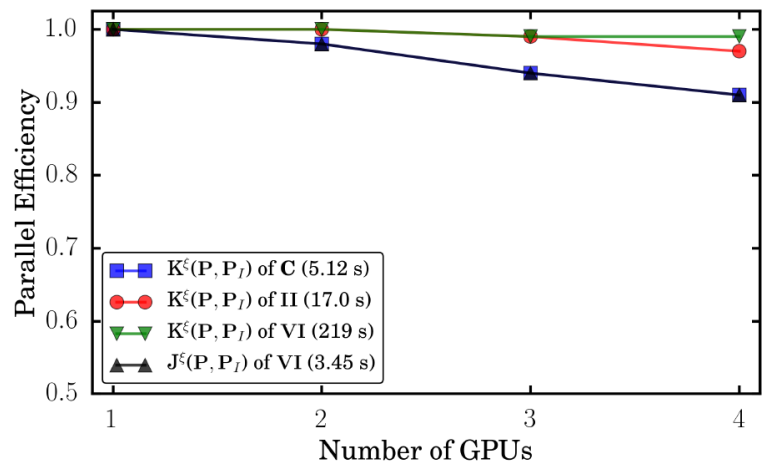

Figure 4. Parallel efficiency of selected GPU integral routines for the calculations of C, II, and VI. The computational time of the integral evaluation using four GPUs is given in parentheses. For details on the calculations and the computational setup, see Section 3.

slowly at the PBE/def2-TZVP level of theory (seven instead of four iterations).

When comparing CPU and GPU timings of the entire calculations, we observe speed-ups of three and eight for VI and VII, respectively. This clearly shows that the use of GPUs becomes more attractive with increasing system size, where the integral evaluations dominate the overall computational time. However, even for relatively small molecule II we already obtain a speed-up of two. These speed-ups exclusively stem from the Coulomb and exchange calculations as the linear algebra and the evaluation of the exchange-correlation kernels are performed entirely on CPUs. The entire calculations of II and VII at the PBE0/def2-SVP level of theory take $\sim 5 \mathrm{~min}$ and $\sim 5 \mathrm{~h}$, respectively, enabling NAMD simulations of II and the calculation of excited-state properties of VII on a reasonable time scale and yet good accuracy.

4.4. Scaling with the Computational Resources. In Figure 4, we show the parallel efficiency of $J / K\left(\mathbf{X}_{I}+\mathbf{Y}_{I}\right)$ (Figure $4 \mathrm{a}$ ) and $J^{\xi} / K^{\xi}\left(\mathbf{P}, \mathbf{P}_{I}\right.$ ) (Figure $4 \mathrm{~b}$ ) for II, VI, and rotary molecular machine $\mathbf{C}\left(\mathrm{C}_{10} \mathrm{H}_{13} \mathrm{FN}\right)$, which will be investigated 
in the next section using up to four GPUs. The Coulomb integrals of II and $\mathbf{C}$ are not analyzed because their computational time is too short.

Figure 4 again shows the suitability of GPUs for large molecular systems. For the time-consuming integral evaluations of VI, we observe a nearly perfect scaling of $>0.9$. This also indicates that adding even more GPUs will still lead to decent speed-ups of the calculations. The parallel efficiency observed in the case of the smaller molecules is lower but still remarkable considering that some computational times are $<1 \mathrm{~s}$. This strong scaling makes the use of GPUs also attractive for small to medium-sized molecules, as shown in the rotary molecular machine example presented in this work.

\section{ILLUSTRATIVE EXAMPLES: ROTARY MOLECULAR MACHINES}

As a prototypical example, we investigate the properties and the dynamics of four newly designed rotary molecular machines. For structures and definitions, see Figure 5. Similar to previous ab initio studies, ${ }^{41-44}$ our machines contain a $\mathrm{C}=$ $\mathrm{N}^{+}$motif, which is also present in the chromophore of rhodopsin. Upon excitation, the molecule should rotate around the central $\mathrm{C}-\mathrm{C}$ double bond. The fluoride substituent should accelerate this rotation because of the steric repulsion, whereas the puckering of the six-membered ring should influence the direction of the rotation. ${ }^{43,44}$ Here, we want to investigate the influence of the atom or group $\mathrm{X}$ adjacent to the central double bond on the light-driven rotation of the molecule. We use $\mathrm{CH}_{2}, \mathrm{NH}, \mathrm{S}$, and $\mathrm{O}$ as $\mathrm{X}$ denoted as $\mathbf{C}, \mathbf{N}, \mathbf{S}$, and $\mathbf{O}$, respectively.

When calculating the excited-state properties and dynamics of the rotary molecular machines, we switch to the TammDancoff approximation (TDA). ${ }^{63}$ As discussed in Section 2.2, this accelerates the calculation and leads to more stable trajectories close to conical intersections. ${ }^{39,64}$ A comparison of the excited-state energies and properties at the TDA and RPA levels of theory is presented in the Supporting Information, where we show mean differences as well as plots of the difference density, the first excited-state gradient, and the NACV between the ground state and the first excited state. We observe an average difference below $10^{-2}$ a.u., with the state ordering and the shape of relaxed difference densities not being affected. The comparison of TDA and CASSCF energies (Supporting Information) also proves the suitability of TDA for the investigated problem. Trends between the systems and the energies close to the conical intersection agree remarkably well. Moreover, we apply the looser accurate thresholds $\left(\vartheta_{\text {pre }}=\right.$ $10^{-3}, \vartheta_{\text {pre }}^{\nabla}=10^{-10}$, and $\left.\vartheta_{\text {TDDFT }}=10^{-5}\right)$ because they introduce only a maximum average error of a few $10^{-5}$ a.u. (Supporting (a)

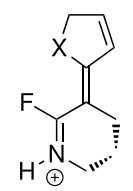

(b)

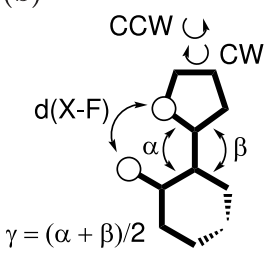

Figure 5. (a) Structures of rotary molecular machines $\mathbf{C}, \mathbf{N}, \mathbf{S}$, and $\mathbf{O}$ and (b) definitions of the $\mathrm{X}-\mathrm{F}$ distance $(d(\mathrm{X}-\mathrm{F}))$, the dihedral angle $(\gamma)$, and the direction of rotation: clockwise $(\mathrm{CW})$ and counterclockwise (CCW).

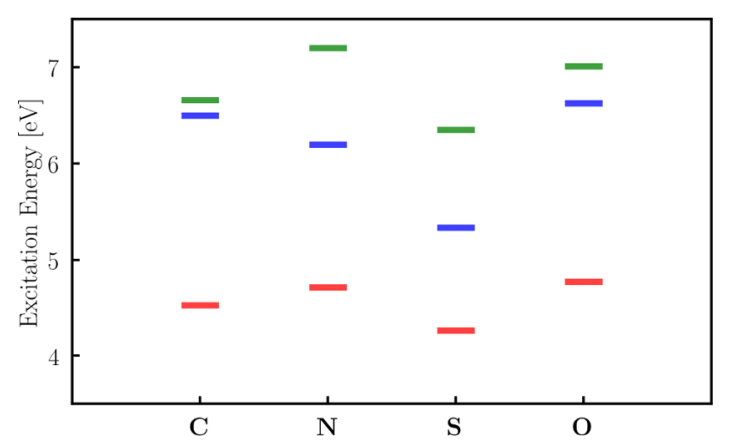

Figure 6. Singlet excitation energies of the four rotary molecular machines (C, N, S, and $\mathbf{O}$ ) calculated at the TDA (wB97/def2-SVP) level of theory. For details on the calculations, see Section 3.3.

Information). Additional details on the calculations are listed in Section 3.3.

In Figure 6, we show the excitation energies of $\mathbf{C}, \mathbf{N}, \mathbf{S}$, and O. Changing the substituent has an influence on the bright $S_{1}$ state of the rotary molecular motor. While the difference density plots look similar for all molecules, the excitation energies increase $(\mathbf{S}<\mathbf{C}<\mathbf{N}<\mathbf{O})$ and the directions of the gradients and NACVs change slightly. This is due to the different electronegativities of the substituents $(\mathrm{C} \approx \mathrm{S}<\mathrm{N}<$ $\mathrm{O}$ ) and the fact that $\mathrm{S}, \mathrm{N}$, and $\mathrm{O}$ atoms have free electron pairs.

To study their effect on the light-induced rotation, we conducted NAMD simulations (105 trajectories for each rotor). In Table 3, we list the percentage of the trajectories that showed a rotation $(\eta)$ as well as the ratio of clockwise and counterclockwise rotations $(r)$. The direction of the rotation has been determined using the dihedral $\gamma$. (See the Supporting Information for plots.) The time-dependent occupation of the $\mathrm{S}_{1}$ state (calculated as an average over all trajectories) and $d(\mathrm{X}-\mathrm{F})$ for the trajectory with the fastest rotation of $\mathbf{C}, \mathbf{N}, \mathbf{S}$, and $\mathbf{O}$, respectively, are shown in Figure 7 .

For all system, we observed (1) the expected rotation around the central $\mathrm{C}-\mathrm{C}$ bond, which can be detected using either $\gamma$ or $d(\mathrm{X}-\mathrm{F})$ and (2) the relaxation from $S_{1}$ to the ground state $\left(S_{0}\right)$. Movies of the rotations are available at https://www.cup.uni-muenchen.de/pc/ochsenfeld/download/ . The decay of the $S_{1}$ population (Figure 7a) seems to correlate with the rotational speed of the corresponding rotary molecular machine (Figure $7 \mathrm{~b}$ ). C shows the fastest rotation and the fastest decay, followed by $\mathbf{S}$ and $\mathbf{O}$, which behave nearly identically. The rotation of $\mathbf{N}$ is the slowest, so is the decay of the $S_{1}$ occupancy. The trend in the rotational speeds $(\mathbf{N}<\mathbf{S}<\mathbf{O}<\mathbf{C})$ can easily be explained. $\mathbf{O}$ rotates slower than $\mathrm{C}$ because the nuclear repulsion between $\mathrm{X}$ and $\mathrm{F}$ is smaller. In the case of $S$, the repulsion of the $S$ atom should be similar to that of the $\mathrm{CH}_{2}$ moiety, but the time for the rotation increases because of its larger mass. The rotation of $\mathbf{N}$ is even slower because of the hydrogen bond between the $\mathrm{NH}$ moiety and the $\mathrm{F}$ atom.

However, this trend cannot be observed when looking at the rotational efficiency ( $\eta$, see Table 3 ). Although $\mathbf{C}$ shows the largest number of trajectories featuring a rotation (70\%), the $\eta$ of $\mathbf{S}$ and $\mathbf{O}(\sim 55 \%)$ is significantly smaller than the $\eta$ of $\mathbf{N}$ (61\%). The reason for this might be the slightly different coupling vectors between the ground state and the first excited state (Supporting Information). In contrast to refs 43 and 44, we do not observe an influence of the puckering of the ring on the rotation. The ratios of $\mathrm{CW}$ and $\mathrm{CCW}$ rotations $(r)$ are 
Table 3. Efficiency ( $\boldsymbol{\eta}$, Determined from the Percentage of Rotating Molecules in the NAMD Simulations) of the Four Rotary Molecular Machines (C, N, S, and O) and Ratio $[r=$ $n(\mathrm{CW}) / n(\mathrm{CCW})]$ of $\mathrm{CW}$ and CCW Rotations

\begin{tabular}{ccccc} 
& $\mathbf{C}$ & $\mathbf{N}$ & $\mathbf{S}$ & $\mathbf{O}$ \\
$\eta$ & $70 \%$ & $61 \%$ & $54 \%$ & $56 \%$ \\
$r$ & 0.68 & 0.78 & 1.19 & 1.68 \\
\hline
\end{tabular}

close to 1 for all four rotors, and no general trend is visible in the $\gamma$ plots (Supporting Information).

Changing $\mathrm{X}$ thus has an interesting effect on the rotary molecular machines. While the excitation energy changes significantly, the ability of the molecule to rotate around the central $\mathrm{C}-\mathrm{C}$ bond is (nearly) preserved. These systems may thus be a good starting point for the design of a series of molecular rotors with tunable excitation energy. Our GPUbased routines aide these investigations by accelerating NAMD simulations even for these small systems. Because of the strong scaling (shown in Figure 4), we observe speed-ups in Coulomb and exchange integral evaluations (even when their computing time is shorter than $1 \mathrm{~s}$ ), leading to a total speed-up of two with respect to a calculation entirely on CPUs. One trajectory took $\sim 1$ day on two Intel Xeon CPU E5 2640 v4@ $2.20 \mathrm{GHz}$ (20 threads) CPUs and four AMD FirePro 3D W8100 GPUs.

\section{CONCLUSIONS}

Throughout this work, we have examined the use of graphics processing units (GPUs) for the evaluation of two-electron integrals in excited-state energies and property (gradients and nonadiabatic coupling vectors) calculations at the timedependent density functional theory (TDDFT) level of theory. Similar to ground-state calculations, we observe that the use of GPUs along with the J-engine ${ }^{73,74}$ and the preLinK scheme ${ }^{50,51}$ leads to decent speed-ups of the integral calculations while additionally showing a reduced scaling behavior depending on the locality of the examined excitation. These speed-ups may even become larger with our currently developed seminumerical exchange scheme. ${ }^{95}$ By using GPUs, nonadiabatic molecular dynamics become more efficient for large but also small molecules (as the a result of the strong scaling) without a loss of accuracy or the introduction of further assumptions. As a first example, we investigated a series of newly designed rotary molecular machines showing that one can tune the excitation energy of these systems without losing their ability to rotate by changing the hetereocycle. For future applications, we consider extending the current implementation toward decoherence corrections ${ }^{96}$ and triplet states. ${ }^{97}$

\section{NOTATION}

All equations in this section use the same notation as in Section 2. $g_{\mathrm{xc}}$ are the third order exchange correlation functional derivatives. The linear transformations $\mathrm{H}^{+}$and $\mathrm{H}^{-}$ are defined as follows:

$$
\begin{gathered}
H_{p q}^{+}[\mathbf{V}]=\sum_{r s}\left\{2(p q \mid r s)+2 f_{p q r s}^{x c}-c_{x}[(p s \mid r q)-(p r \mid s q)]\right\} V_{r s} \\
H_{p q}^{-}[\mathbf{V}]=\sum_{r s}\left\{c_{\mathbf{X}}[(p s \mid r q)-(p r \mid s q)]\right\} V_{r s}
\end{gathered}
$$

\section{EXCITED-STATE ENERGY GRADIENTS}

The unrelaxed difference density matrix $\mathbf{T}$ is calculated as follows:

$$
\mathbf{T}_{I}=\frac{1}{2}\left(\begin{array}{cc}
-\left(\mathbf{R}_{I}^{T} \mathbf{R}_{I}+\mathbf{L}_{I}^{T} \mathbf{L}_{I}\right)_{i j} & 0 \\
0 & \left(\mathbf{R}_{I} \mathbf{R}_{I}^{T}+\mathbf{L}_{I} \mathbf{L}_{I}^{T}\right)_{a b}
\end{array}\right)
$$

To obtain the Lagrangian multiplier $\mathbf{Z}_{I}$ it is necessary to solve the following $\mathrm{Z}$-vector equation:

$$
\begin{aligned}
& \sum_{j b}(\mathbf{A}+\mathbf{B})_{i j a b} Z_{j b}^{I}=-U_{i a}^{I} \\
& U_{i a}^{I}=\sum_{b}\left\{R_{i b}^{I} H_{a b}^{+}\left[\mathbf{R}_{I}\right]+L_{i b}^{I} H_{a b}^{-}\left[\mathbf{L}_{I}\right]\right\} \\
& \quad-\sum_{j}\left\{R_{j a}^{I} H_{j i}^{+}\left[\mathbf{R}_{I}\right]+L_{j a}^{I} H_{j i}^{-}\left[\mathbf{L}_{I}\right]\right\}+H_{i a}^{+}\left[\mathbf{T}_{I}\right] \\
& \quad+2 \sum_{j b k c}\left\{g_{i a j b k c}^{x c} R_{j b}^{I} R_{k c}^{I}\right\}
\end{aligned}
$$

Solving this $\mathrm{Z}$-vector equation thus leads to the calculation of $\mathbf{Z}_{I}$, which is used to determine the relaxed difference density matrix $\left(\mathbf{P}_{I}\right)$ :

$$
\mathbf{P}_{I}=\mathbf{T}_{I}+\mathbf{Z}_{I}
$$

(a)

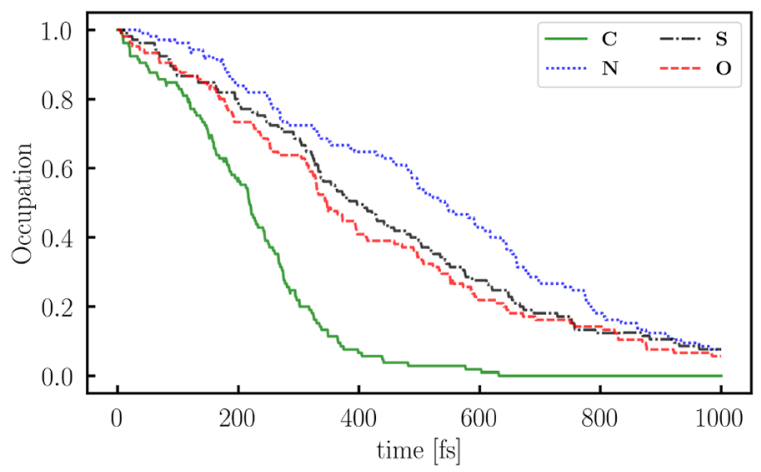

(b)

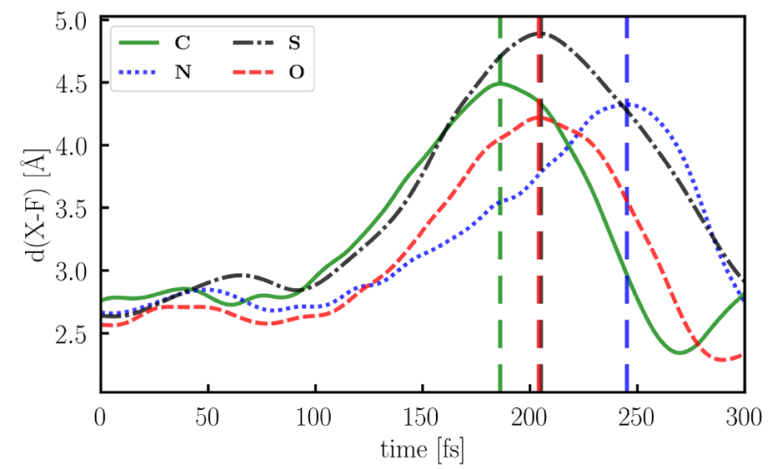

Figure 7. (a) Time-dependent decay of the occupancy of the $S_{1}$ state determined as a mean of all nonadiabatic molecular dynamics simulations. (b) Change in $d(\mathrm{X}-\mathrm{F})$ during one selected trajectory of $\mathbf{C}, \mathbf{N}, \mathbf{S}$, and $\mathbf{O}$, respectively, showing the rotation of the molecule around the central $\mathrm{C}-\mathrm{C}$ bond. The maxima $\left(180^{\circ}\right.$ rotation) are marked by the vertical dashed lines. 
Using the calculated matrices, the energy-weighted difference density matrix $\left(\mathbf{W}_{I}\right)$ can be formed:

$$
\begin{gathered}
W_{i j}^{I}=\sum_{a} \omega_{I}\left\{R_{i a}^{I} L_{j a}^{I}+L_{i a}^{I} R_{j a}^{I}\right\} \\
-\sum_{a} \epsilon_{a}\left\{R_{i a}^{I} R_{j a}^{I}+L_{i a}^{I} L_{j a}^{I}\right\} \\
+H_{i j}^{+}\left[\mathbf{P}_{I}\right]+2 \sum_{k c l d}\left\{g_{i j k c l d}^{\mathrm{xc}} R_{k c}^{I} R_{l d}^{I}\right\} \\
W_{a b}^{I}=\sum_{i} \omega_{I}\left\{R_{i a}^{I} L_{i b}^{I}+L_{i a}^{I} R_{i b}^{I}\right\} \\
+\sum_{i} \epsilon_{i}\left\{R_{i a}^{I} R_{i b}^{I}+L_{i a}^{I} L_{i b}^{I}\right\} \\
W_{i a}^{I}=\sum_{j}\left\{R_{j a}^{I} H_{j i}^{+}\left[\mathbf{R}_{I}\right]+L_{j a}^{I} H_{j i}^{-}\left[\mathbf{L}_{I}\right]\right\}+\epsilon_{i} Z_{i a}^{I}
\end{gathered}
$$

The calculation of the effective two-particle difference density matrix is

$$
\begin{aligned}
& \Gamma_{\mu \nu \kappa \lambda}^{I}=\frac{1}{2}\left\{2 P_{\mu \nu}^{I} D_{\kappa \lambda}+2 R_{\mu \nu}^{I} R_{\kappa \lambda}^{I}-c_{\mathrm{x}}\left[P_{\mu \lambda}^{I} P_{\kappa \nu}+P_{\mu \kappa}^{I} P_{\lambda \nu}\right.\right. \\
& \left.\left.\quad+R_{\mu \lambda}^{I} R_{\kappa \nu}^{I}+R_{\mu \kappa}^{I} R_{\lambda \nu}^{I}-L_{\mu \lambda}^{I} L_{\kappa \nu}^{I}+L_{\mu \kappa}^{I} L_{\lambda \nu}^{I}\right]\right\}
\end{aligned}
$$

\section{NONADIABATIC COUPLING VECTORS BETWEEN} THE GROUND AND AN EXCITED STATE

First the Lagrangian multipliers $\mathbf{Z}_{0 I}$ and $\mathbf{W}_{0 I}$ need to be determined. In order to obtain $\mathrm{Z}_{0 I}$ the following $\mathrm{Z}$-vector equation has to be solved:

$$
\sum_{j b}(\mathbf{A}+\mathbf{B})_{i j a b} Z_{j b}^{0 I}=L_{i a}^{I}
$$

An iterative solution of eq 26 is not necessary. $\mathbf{Z}_{0 I}$ is equal to $\mathbf{R}_{I}$ and the relaxed difference density matrix $\left(\mathbf{P}_{0 I}\right)$ is calculated as follows:

$$
\mathbf{P}_{0 I}=\mathbf{Z}_{0 I}=\frac{1}{\omega_{I}} \mathbf{R}_{I}
$$

The calculation of $\mathbf{W}_{0 I}$ is presented in eq 28 .

$$
\begin{gathered}
W_{i j}^{0 I}=H_{i j}^{+}\left[\mathbf{P}_{0 I}\right], \\
W_{a b}^{0 I}=0, \\
W_{i a}^{0 I}=\frac{1}{2} L_{i a}^{I}+\epsilon_{i} P_{i a}^{0 I}
\end{gathered}
$$

$\Gamma_{0 I}$ is defined as

$$
\Gamma_{\mu \nu \kappa \lambda}^{0 I}=\frac{1}{2}\left(2 P_{\mu \nu}^{0 I} P_{\kappa \lambda}-c_{\mathrm{x}}\left[P_{\mu \lambda}^{0 I} P_{\kappa \nu}+P_{\nu \lambda}^{0 I} P_{\kappa \mu}\right]\right)
$$

NONADIABATIC COUPLING VECTORS BETWEEN TWO EXCITED STATES

The unrelaxed difference density matrix $\left(\mathbf{T}_{I J}\right)$ is calculated as follows:

$$
\begin{gathered}
T_{i j}^{I J}=-\frac{1}{4}\left(\mathbf{R}_{I}^{T} \mathbf{R}_{J}+\mathbf{R}_{J}^{T} \mathbf{R}_{I}+\mathbf{L}_{I}^{T} \mathbf{L}_{J}+\mathbf{L}_{J}^{T} \mathbf{L}_{I}\right)_{i j}, \\
T_{a b}^{I J}=\frac{1}{4}\left(\mathbf{R}_{I} \mathbf{R}_{J}^{T}+\mathbf{R}_{J} \mathbf{R}_{I}^{T}+\mathbf{L}_{I} \mathbf{L}_{J}^{T}+\mathbf{L}_{J} \mathbf{L}_{I}^{T}\right)_{a b}
\end{gathered}
$$

To obtain the Lagrangian multiplier $\mathbf{Z}_{I J}$ it is necessary to solve the following $\mathrm{Z}$-vector equation:

$$
\begin{aligned}
& \sum_{j b}(\mathbf{A}+\mathbf{B})_{i j a b} Z_{j b}^{I J}=-U_{i a}^{I J} \\
& U_{i a}^{I J}=\frac{1}{2} \sum_{b}\left\{R_{i b}^{I} H_{a b}^{+}\left[\mathbf{R}_{J}\right]+L_{i b}^{I} H_{a b}^{-}\left[\mathbf{L}_{J}\right]\right\} \\
& \quad+\frac{1}{2} \sum_{b}\left\{R_{i b}^{J} H_{a b}^{+}\left[\mathbf{R}_{I}\right]+L_{i b}^{J} H_{a b}^{-}\left[\mathbf{L}_{I}\right]\right\} \\
& \quad-\frac{1}{2} \sum_{j}\left\{R_{j a}^{I} H_{j i}^{+}\left[\mathbf{R}_{J}\right]+L_{j a}^{I} H_{j i}^{-}\left[\mathbf{L}_{J}\right]\right\} \\
& \quad-\frac{1}{2} \sum_{j}\left\{R_{j a}^{J} H_{j i}^{+}\left[\mathbf{R}_{I}\right]+L_{j a}^{J} H_{j i}^{-}\left[\mathbf{L}_{I}\right]\right\}+H_{i a}^{+}\left[\mathbf{T}_{I J}\right] \\
& \quad+2 \sum_{j b k c}\left\{g_{i a j b k c}^{\mathrm{xc}} R_{j b}^{I} R_{k c}^{J}\right\}
\end{aligned}
$$

$\mathbf{Z}_{I J}$ is used to calculate the relaxed difference density matrix $\left(\mathbf{P}_{I J}\right)$ :

$$
\mathbf{P}_{I J}=\mathbf{T}_{I J}+\mathbf{Z}_{I J}
$$

The calculation of $\mathbf{W}_{I J}$ is

$$
\begin{aligned}
& W_{i j}^{I J}=-\frac{1}{4} \sum_{a} \varepsilon_{a}\left\{R_{i a}^{I} R_{j a}^{J}+L_{i a}^{I} L_{j a}^{J}+\right. \\
& \left.R_{i a}^{J} R_{j a}^{I}+L_{i a}^{J} L_{j a}^{I}\right\} \\
& +H_{i j}^{+}\left[\mathbf{P}_{I J}\right]+2 \sum_{k c l d}\left\{g_{i j k c l d}^{\mathrm{xc}} R_{k c}^{I} R_{l d}^{J}\right\} \\
& -\frac{1}{2} \sum_{a} \omega_{I}\left\{R_{i a}^{I} L_{j a}^{J}+L_{i a}^{I} R_{j a}^{J}\right\} \\
& -\frac{1}{2} \sum_{a} \omega_{J}\left\{R_{i a}^{J} L_{j a}^{I}+L_{i a}^{J} R_{j a}^{I}\right\} \\
& W_{a b}^{I J}=\frac{1}{4} \sum_{i} \varepsilon_{i}\left\{R_{i a}^{I} R_{i b}^{J}+L_{i a}^{I} L_{i b}^{J}+\right. \\
& \left.R_{i a}^{J} R_{i b}^{I}+L_{i a}^{J} L_{i b}^{I}\right\} \\
& +\frac{1}{2} \sum_{i} \omega_{I}\left\{R_{i a}^{I} L_{i b}^{J}+L_{i a}^{I} R_{i b}^{J}\right\} \\
& +\frac{1}{2} \sum_{i} \omega_{J}\left\{R_{i a}^{J} L_{i b}^{I}+L_{i a}^{J} R_{i b}^{I}\right\} \\
& W_{i a}^{I J}=\frac{1}{2} \sum_{j}\left\{R_{j a}^{I} H_{j i}^{+}\left[\mathbf{R}_{J}\right]+L_{j a}^{I} H_{j i}^{-}\left[\mathbf{L}_{J}\right]\right\} \\
& +\frac{1}{2} \sum_{j}\left\{R_{j a}^{J} H_{j i}^{+}\left[\mathbf{R}_{I}\right]+L_{j a}^{J} H_{j i}^{-}\left[\mathbf{L}_{I}\right]\right\}+\varepsilon_{i} Z_{i a} .
\end{aligned}
$$

$\Gamma_{I J}$ is defined as 


$$
\begin{aligned}
\Gamma_{\mu \nu \kappa \lambda}^{I J}= & \frac{1}{2}\left\{2 P_{\mu \nu}^{I J} P_{\kappa \lambda}+2 R_{\mu \nu}^{I} R_{\kappa \lambda}^{J}-c_{\mathrm{x}}\left[P_{\mu \lambda}^{I J} P_{\kappa \nu}+P_{\mu \kappa}^{I J} P_{\lambda \nu}\right.\right. \\
& \left.\left.+R_{\mu \lambda}^{I} R_{\kappa \nu}^{J}+R_{\mu \kappa}^{I} R_{\lambda \nu}^{J}-L_{\mu \lambda}^{I} L_{\kappa \nu}^{J}+L_{\mu \kappa}^{I} L_{\lambda \nu}^{J}\right]\right\}
\end{aligned}
$$

\section{ASSOCIATED CONTENT}

\section{S Supporting Information}

The Supporting Information is available free of charge at https://pubs.acs.org/doi/10.1021/acs.jctc.9b00859.

$\log -\log$ plots of the integral timings for the determination of the scaling behavior; validation of the use of the TDA and accurate thresholds, with excited-state energies, relaxed difference density plots, and plots of excited-state gradients and nonadiabatic coupling vectors at the TDA and RPA levels of theory; behavior of the dihedral $\gamma$ during all trajectories (PDF)

\section{AUTHOR INFORMATION}

\section{Corresponding Author}

*E-mail: c.ochsenfeld@fkf.mpg.de.

\section{ORCID}

Christian Ochsenfeld: 0000-0002-4189-6558

Notes

The authors declare no competing financial interest.

\section{ACKNOWLEDGMENTS}

We thank Gökçen Savaşçi for providing the optimized structures of the covalent organic framework pores (VI and VII) and Thomas Schnappinger and Prof. Regina de VivieRiedle (LMU Munich) for helpful discussions. Financial support was provided by the Innovative Training Network "Computational Spectroscopy in Natural Sciences and Engineering" (ITN-COSINE) and the DFG cluster of excellence (EXC 2089) “e-conversion”. C.O. acknowledges further support as a Max-Planck Fellow at the MPI-FKF Stuttgart.

\section{REFERENCES}

(1) Turro, N. J. Modern Molecular Photochemistry; University Science Books: Sausalito, CA, 1991.

(2) Avouris, P.; Gelbart, W. M.; El-Sayed, M. A. Nonradiative electronic relaxation under collision-free conditions. Chem. Rev. 1977, 77, 793-833.

(3) May, V.; Kühn, O. Charge and Energy Transfer Dynamics in Molecular Science; Wiley-VCH: Weinheim, 2005.

(4) Lozier, R. H.; Bogomolni, R. A.; Stoeckenius, W. Bacteriorhodopsin: a light-driven proton pump in Halobacterium Halobium. Biophys. J. 1975, 15, 955-962.

(5) Koumura, N.; Zijlstra, R. W. J.; van Delden, R. A.; Harada, N.; Feringa, B. L. Light-driven monodirectional molecular rotor. Nature 1999, 401, 152-155.

(6) Guentner, M.; Schildhauer, M.; Thumser, S.; Mayer, P.; Stephenson, D.; Mayer, P. J.; Dube, H. Sunlight-powered kHz rotation of a hemithioindigo-based molecular motor. Nat. Commun. 2015, 6, 8406.

(7) Astumian, R. D. How molecular motors work-insights from the molecular machinist's toolbox: the Nobel prize in Chemistry 2016 . Chem. Sci. 2017, 8, 840-845.

(8) Dreuw, A. Quantum chemical methods for the investigation of photoinitiated processes in biological systems: Theory and applications. ChemPhysChem 2006, 7, 2259-2274.

(9) Roos, B. A new method for large-scale $\mathrm{Cl}$ calculations. Chem. Phys. Lett. 1972, 15, 153-159.
(10) Trofimov, A. B.; Krivdina, I. L.; Weller, J.; Schirmer, J. Algebraic-diagrammatic construction propagator approach to molecular response properties. Chem. Phys. 2006, 329, 1-10.

(11) Christiansen, O.; Koch, H.; Jörgensen, P. The second-order approximate coupled cluster singles and doubles model CC2. Chem. Phys. Lett. 1995, 243, 409-418.

(12) Casida, M. E. Time-Dependent Density Functional Response Theory for Molecules. In Recent Advances in Density Functional Methods; Chong, D. P., Ed.; Recent Advances in Computational Chemistry; World Scientific, 1995; Part 1, pp 155-192.

(13) Casida, M. E. Time-dependent density-functional theory for molecules and molecular solids. J. Mol. Struct.: THEOCHEM 2009, 914, 3-18.

(14) Dreuw, A.; Head-Gordon, M. Failure of time-dependent density functional theory for long-range charge-transfer excited states: The Zincbacteriochlorin-Bacteriochlorin and BacteriochlorophyllSpheroidene complexes. J. Am. Chem. Soc. 2004, 126, 4007-4016.

(15) Dreuw, A.; Head-Gordon, M. Single-reference ab initio methods for the calculation of excited states of large molecules. Chem. Rev. 2005, 105, 4009-4037.

(16) Laurent, A. D.; Jacquemin, D. TD-DFT benchmarks: A review. Int. J. Quantum Chem. 2013, 113, 2019-2039.

(17) Furche, F.; Ahlrichs, R. Adiabatic time-dependent density functional methods for excited state properties. J. Chem. Phys. 2002, 117, 7433-7447.

(18) Chernyak, V.; Mukamel, S. Density-matrix representation of nonadiabatic couplings in time-dependent density functional (TDDFT) theories. J. Chem. Phys. 2000, 112, 3572.

(19) Baer, R. Non-adiabatic couplings by time-dependent density functional theory. Chem. Phys. Lett. 2002, 364, 75-79.

(20) Hu, C.; Hirai, H.; Sugino, O. Nonadiabatic couplings from time-dependent density functional theory: Formulation in the Casida formalism and practical scheme within modified linear response. $J$. Chem. Phys. 2007, 127, No. 064103.

(21) Tavernelli, I.; Tapavicza, E.; Rothlisberger, U. Non-adiabatic dynamics using time-dependent density functional theory: Assessing the coupling strengths. J. Mol. Struct.: THEOCHEM 2009, 914, 2229.

(22) Tavernelli, I.; Tapavicza, E.; Rothlisberger, U. Nonadiabatic coupling vectors within linear response time-dependent density functional theory. J. Chem. Phys. 2009, 130, 124107.

(23) Send, R; Furche, F. First-order nonadiabatic couplings from time-dependent hybrid density functional response theory: Consistent formalism, implementation, and performance. J. Chem. Phys. 2010, 132, No. 044107.

(24) Tavernelli, I.; Curchod, B. F. E.; Laktionov, A.; Rothlisberger, U. Nonadiabatic coupling vectors for excited states within timedependent density functional theory in the Tamm-Dancoff approximation and beyond. J. Chem. Phys. 2010, 133, 194104.

(25) Fatehi, S.; Alguire, E.; Shao, Y.; Subotnik, J. E. Analytic derivative couplings between configuration-interaction-singles states with built-in electron-translation factors for translational invariance. $J$. Chem. Phys. 2011, 135, 234105.

(26) Ou, Q.; Fatehi, S.; Alguire, E.; Shao, Y.; Subotnik, J. E. Derivative couplings between TDDFT excited states obtained by direct differentiation in the Tamm-Dancoff approximation. J. Chem. Phys. 2014, 141, No. 024114.

(27) Alguire, E. C.; Ou, Q.; Subotnik, J. E. Calculating derivative couplings between time-dependent Hartree-Fock excited states with pseudo-wavefunctions. J. Phys. Chem. B 2015, 119, 7140-7149.

(28) Subotnik, J. E.; Alguire, E. C.; Ou, Q.; Landry, B. R.; Fatehi, S. The requisite electronic structure theory to describe photoexcited nonadiabatic dynamics: Nonadiabatic derivative couplings and diabatic electronic couplings. Acc. Chem. Res. 2015, 48, 1340-1350.

(29) Ou, Q.; Alguire, E. C.; Subotnik, J. E. Derivative couplings between time-dependent density functional theory excited states in the random-phase approximation based on pseudo-wavefunctions: Behavior around conical intersections. J. Phys. Chem. B 2015, 119, 7150-7161. 
(30) Tully, J. C. Molecular dynamics with electronic transitions. J. Chem. Phys. 1990, 93, 1061-1071.

(31) Hammes-Schiffer, S.; Tully, J. C. Proton transfer in solution: Molecular dynamics with quantum transitions. J. Chem. Phys. 1994, 101, 4657-4667.

(32) Wang, L.; Akimov, A.; Prezhdo, O. V. Recent Progress in Surface Hopping: 2011-2015. J. Phys. Chem. Lett. 2016, 7, 2100.

(33) Crespo-Otero, R.; Barbatti, M. Recent Advances and Perspectives on Nonadiabatic Mixed Quantum-Classical Dynamics. Chem. Rev. 2018, 118, 7026.

(34) Mai, S.; Marquetand, P.; González, L. Nonadiabatic Dynamics: The SHARC Approach. WIREs Comput. Mol. Sci. 2018, 8, No. e1370. (35) Tapavicza, E.; Tavernelli, I.; Rothlisberger, U. Trajectory surface hopping within linear response time-dependent densityfunctional theory. Phys. Rev. Lett. 2007, 98, No. 023001.

(36) Barbatti, M.; Granucci, G.; Persico, M.; Ruckenbauer, M.; Vazdar, M.; Eckert-Maksić, M.; Lischka, H. The on-the-fly surfacehopping program system Newton-X: Application to ab initio simulation of the nonadiabatic photodynamics of benchmark systems. J. Photochem. Photobiol., A 2007, 190, 228-240.

(37) Barbatti, M. Nonadiabatic dynamics with trajectory surface hopping method. Wiley Interdiscip. Rev. Comput. Mol. Sci. 2011, 1, 620-633.

(38) Tully, J. C. Perspective: Nonadiabatic dynamics theory. J. Chem. Phys. 2012, 137, 22A301.

(39) Tapavicza, E.; Bellchambers, G. D.; Vincent, J. C.; Furche, F. $\mathrm{Ab}$ initio non-adiabatic molecular dynamics. Phys. Chem. Chem. Phys. 2013, 15, 18336-18348.

(40) Plasser, F.; Crespo-Otero, R.; Pederzoli, M.; Pittner, J.; Lischka, H.; Barbatti, M. Surface hopping dynamics with correlated singlereference methods: $9 \mathrm{H}$-adenine as a case study. J. Chem. Theory Comput. 2014, 10, 1395-1405.

(41) Nikiforov, A.; Gamez, J. A.; Thiel, W.; Filatov, M. Computational Design of a Family of Light-Driven Rotary Molecular Motors with Improved Quantum Efficiency. J. Phys. Chem. Lett. 2016, 7, 105110.

(42) Kazaryan, A.; Lan, Z.; Schäfer, L. V.; Thiel, W.; Filatov, M. Surface hopping excited-state dynamics study of the photoisomerization of a light-driven fluorene molecular rotary motor. J. Chem. Theory Comput. 2011, 7, 2189-2199.

(43) Oruganti, B.; Wang, J.; Durbeej, B. Quantum chemical design of rotary molecular motors. Int. J. Quantum Chem. 2018, 118, No. e25405.

(44) Wang, J.; Oruganti, B.; Durbeej, B. Light-driven rotary molecular motors without point chirality: A minimal design. Phys. Chem. Chem. Phys. 2017, 19, 6952-6956.

(45) Sisto, A.; Glowacki, D. R.; Martinez, T. J. Ab initio nonadiabatic dynamics of multichromophore complexes: A scalable graphicalprocessing-unit-accelerated exciton framework. Acc. Chem. Res. 2014, $47,2857-2866$

(46) Yasuda, K. Two-electron integral evaluation on the graphics processor unit. J. Comput. Chem. 2008, 29, 334-342.

(47) Ufimtsev, I. S.; Martínez, T. J. Quantum chemistry on graphical processing units. 1. strategies for two-electron integral evaluation. J. Chem. Theory Comput. 2008, 4, 222-231.

(48) Ufimtsev, I. S.; Martínez, T. J. Quantum chemistry on graphical processing units. 2. direct self-consistent-field implementation. J. Chem. Theory Comput. 2009, 5, 1004-1015.

(49) Ufimtsev, I. S.; Martínez, T. J. Quantum chemistry on graphical processing units. 3. Analytical energy gradients, geometry optimization, and first principles molecular dynamics. J. Chem. Theory Comput. 2009, 5, 2619-2628.

(50) Kussmann, J.; Ochsenfeld, C. Pre-selective screening for matrix elements in linear-scaling exact exchange calculations. J. Chem. Phys. 2013, 138, 134114.

(51) Kussmann, J.; Ochsenfeld, C. Preselective screening for linearscaling exact exchange-gradient calculations for graphics processing units and general strong-scaling massively parallel calculations. J. Chem. Theory Comput. 2015, 11, 918-922.
(52) Kussmann, J.; Ochsenfeld, C. Hybrid CPU/GPU integral engine for strong-scaling ab initio methods. J. Chem. Theory Comput. 2017, 13, 3153-3159.

(53) Peters, L. D. M.; Kussmann, J.; Ochsenfeld, C. Efficient and accurate Born-Oppenheimer molecular dynamics for large molecular systems. J. Chem. Theory Comput. 2017, 13, 5479-5485.

(54) Isborn, C. M.; Luehr, N.; Ufimtsev, I. S.; Martínez, T. J. Excited-state electronic structure with configuration interaction singles and Tamm-Dancoff time-dependent density functional theory on graphical processing units. J. Chem. Theory Comput. 2011, 7, 1814-1823.

(55) Hohenstein, E. G.; Luehr, N.; Ufimtsev, I. S.; Martínez, T. J. An atomic orbital-based formulation of the complete active space selfconsistent field method on graphical processing units. J. Chem. Phys. 2015, 142, 224103.

(56) Snyder, J. W.; Hohenstein, E. G.; Luehr, N.; Martínez, T. J. An atomic orbital-based formulation of analytical gradients and nonadiabatic coupling vector elements for the state-averaged complete active space self-consistent field method on graphical processing units. J. Chem. Phys. 2015, 143, 154107.

(57) Curchod, B. F. E.; Sisto, A.; Martínez, T. J. Ab Initio Multiple Spawning Photochemical Dynamics of DMABN Using GPUs. J. Phys. Chem. A 2017, 121, 265.

(58) Snyder, J. W.; Curchod, B. F. E.; Martínez, T. J. GPUAccelerated State-Averaged Complete Active Space Self-Consistent Field Interfaced with $\mathrm{Ab}$ Initio Multiple Spawning Unravels the Photodynamics of Provitamin $\mathrm{D}_{3}$. J. Phys. Chem. Lett. 2016, 7, 2444.

(59) Hollas, D.; Sištík, L.; Hohenstein, E. G.; Martínez, T. J.; Slavícek, P. Nonadiabatic Ab Initio Molecular Dynamics with the Floating Occupation Molecular Orbital-Complete Active Space Configuration Interaction Method. J. Chem. Theory Comput. 2018, 14, 339.

(60) Filatov, M.; Min, S. K.; Kim, K. W. Direct Nonadiabatic Dynamics by Mixed Quantum-Classical Formalism Connected with Ensemble Density Functional Theory Method: Application to transPenta-2,4-dieniminium Cation. J. Chem. Theory Comput. 2018, 14, 4499.

(61) Bauernschmitt, R.; Ahlrichs, R. Treatment of electronic excitations within the adiabatic approximation of time dependent density functional theory. Chem. Phys. Lett. 1996, 256, 454-464.

(62) Bauernschmitt, R.; Häser, M.; Treutler, O.; Ahlrichs, R. Calculation of excitation energies within time-dependent density functional theory using auxiliary basis set expansions. Chem. Phys. Lett. 1997, 264, 573-578.

(63) Maurice, D.; Head-Gordon, M. Configuration interaction with single substitutions for excited states of open-shell molecules. Int. J. Quantum Chem. 1995, 56, 361-370.

(64) Cordova, F.; Doriol, L. J.; Casida, M. E. Troubleshooting timedependent density-functional theory for photochemical applications: Oxirane. J. Chem. Phys. 2007, 127, 164111.

(65) Chai, J. D.; Head-Gordon, M. Systematic optimization of longrange corrected hybrid density functionals. J. Chem. Phys. 2008, 128, No. 084106.

(66) Li, Z.; Liu, W. First-order nonadiabatic coupling matrix elements between excited states: A Lagrangian formulation at the CIS, RPA, TD-HF, and TD-DFT levels. J. Chem. Phys. 2014, 141, No. 014110.

(67) Li, Z.; Suo, B.; Liu, W. First order nonadiabatic coupling matrix elements between excited states: Implementation and application at the TD-DFT and pp-TDA levels. J. Chem. Phys. 2014, 141, 244105.

(68) Ou, Q.; Bellchambers, G. D.; Furche, F.; Subotnik, J. E. Firstorder derivative couplings between excited states from adiabatic TDDFT response theory. J. Chem. Phys. 2015, 142, No. 064114.

(69) Zhang, X.; Herbert, J. M. Analytic derivative couplings in timedependent density functional theory: Quadratic response theory versus pseudo-wavefunction approach. J. Chem. Phys. 2015, 142, No. 064109.

(70) Parker, S. M.; Roy, S.; Furche, F. Unphysical divergences in response theory. J. Chem. Phys. 2016, 145, 134105. 
(71) Zhang, X.; Herbert, J. M. Analytic derivative couplings for spinflip configuration interaction singles and spin-flip time-dependent density functional theory. J. Chem. Phys. 2014, 141, No. 064104.

(72) Liu, J.; Thiel, W. An efficient implementation of semiempirical quantum-chemical orthogonalization-corrected methods for excitedstate dynamics. J. Chem. Phys. 2018, 148, 154103.

(73) Reza Ahmadi, G.; Almlöf, J. The Coulomb operator in a Gaussian product basis. Chem. Phys. Lett. 1995, 246, 364-370.

(74) White, C.; Head-Gordon, M. A J matrix engine for density functional theory calculations. J. Chem. Phys. 1996, 104, 2620-2629.

(75) intel c/c++ compiler v19.0, see https://software.intel.com.

(76) Marques, M. A. L.; Oliveira, M. J. T.; Burnus, T. Libxc: A library of exchange and correlation functionals for density functional theory. Comput. Phys. Commun. 2012, 183, 2272-2281.

(77) Lehtola, S.; Steigemann, C.; Oliveira, M. J.; Marques, M. A. Recent developments in LIBXC A comprehensive library of functionals for density functional theory. SoftwareX 2018, 7, 1-5.

(78) Laqua, H.; Kussmann, J.; Ochsenfeld, C. An improved molecular partitioning scheme for numerical quadratures in density functional theory. J. Chem. Phys. 2018, 149, 204111.

(79) Morin, J.-F.; Shirai, Y.; Tour, J. M. En Route to a motorized nanocar. Org. Lett. 2006, 8, 1713-1716.

(80) Vyas, V. S.; Haase, F.; Stegbauer, L.; Savasci, G.; Podjaski, F.; Ochsenfeld, C.; Lotsch, B. V. A tunable azine covalent organic framework platform for visible light-induced hydrogen generation. Nat. Commun. 2015, 6, 8508.

(81) Perdew, J. P.; Burke, K.; Ernzerhof, M. Generalized Gradient Approximation made simple. Phys. Rev. Lett. 1996, 77, 3865.

(82) Perdew, J. P.; Burke, K.; Ernzerhof, M. Generalized Gradient Approximation made simple. Phys. Rev. Lett. 1997, 78, 1396.

(83) Ernzerhof, M.; Scuseria, G. E. Assessment of the Perdew-BurkeErnzerhof exchange-correlation functional. J. Chem. Phys. 1999, 110, 5029.

(84) Adamo, C.; Barone, V. Toward reliable density functional methods without adjustable parameters: The PBE0 model. J. Chem. Phys. 1999, 110, 6158.

(85) Weigend, F.; Ahlrichs, A. Balanced basis sets of split valence, triple zeta valence and quadruple zeta valence quality for $\mathrm{H}$ to $\mathrm{Rn}$ : Design and assessment of accuracy. Phys. Chem. Chem. Phys. 2005, 7, 3297.

(86) Weigend, F. Accurate Coulomb-fitting basis sets for H to Rn. Phys. Chem. Chem. Phys. 2006, 8, 1057.

(87) White, C. A.; Head-Gordon, M. Linear and sublinear scaling formation of Hartree-Fock-type exchange matrices. Chem. Phys. Lett. 1994, 230, 8.

(88) White, C. A.; Johnson, B. G.; Gill, P. M. W.; Head-Gordon, M. Linear and sublinear scaling formation of Hartree-Fock-type exchange matrices. Chem. Phys. Lett. 1996, 253, 268.

(89) Ochsenfeld, C.; White, C. A.; Head-Gordon, M. Linear and sublinear scaling formation of Hartree-Fock-type exchange matrices. J. Chem. Phys. 1998, 109, 1663-1669.

(90) Ochsenfeld, C. Linear scaling exchange gradients for HartreeFock and hybrid density functional theory. Chem. Phys. Lett. 2000, 327, 216-223.

(91) Verlet, L. Computer "experiments" on classical fluids. I. Thermodynamical properties of Lennard-Jones molecules. Phys. Rev. 1967, 159, 98-103.

(92) Swope, W. C.; Andersen, H. C.; Berens, P. H.; Wilson, K. R. A computer simulation method for the calculation of equilibrium constants for the formation of physical clusters of molecules: Application to small water clusters. J. Chem. Phys. 1982, 76, 637-649.

(93) Niklasson, A. M. N.; Steneteg, P.; Odell, A.; Bock, N.; Challacombe, M.; Tymczak, C. J.; Holmström, E.; Zheng, G.; Weber, V. Extended Lagrangian Born-Oppenheimer molecular dynamics with dissipation. J. Chem. Phys. 2009, 130, 214109.

(94) Bussi, G.; Donadio, D.; Parrinello, M. Canonical sampling through velocity rescaling. J. Chem. Phys. 2007, 126, No. 014101.

(95) Laqua, H.; Kussmann, J.; Ochsenfeld, C. Submitted for publication, 2019.
(96) Jaeger, H. M.; Fischer, S.; Prezhdo, O. V. Decoherence-induced surface hopping. J. Chem. Phys. 2012, 137, 22 A545.

(97) Richter, M.; Marquetand, P.; González-Vázquez, J.; Sola, I.; González. SHARC: ab Initio Molecular Dynamics with Surface Hopping in the Adiabatic Representation Including Arbitrary Couplings. J. Chem. Theory Comput. 2011, 7, 1253. 